\title{
Über die Veränderungen des intermediären Kohlehydrat= und Wasserstofiwechsels in der Leber in Shockzuständen.
}

\author{
II. Mitteilung: Histamin- und Peptonshock. \\ Von \\ Shigeo Tsuge. \\ (椎椎而头) \\ (Aus der Medizinischen Klinik von Prof: Dr. T. Kato, \\ Tohoku-Reichsuniversität zu Sendai.)
}

In der I. Mitteilung ${ }^{1)}$ habe ich, um die beim anaphylaktischen Shock auftretenden Veränderungen im intermediären Kohlehydrat- sowie Wasserstoffwechsel an der Kaninchenleber zn erforschen, am in die Leber einströmenden arteriellen und Pfortaderblut und am aus derselben ausströmenden Lebervenenblut den Milchsäure-, Zucker-, Hämoglobin- und Serumeiweissgehalt bestimmt. Durch diese Untersuchungen bin ich zur Erkenntnis gelangt, dass sich in der Leber beim anaphylaktischen Shock bestimmte Veränderungen in jedem einzelnen Stoffumsatz abspielen. Nun ist es seit langem allgemein bekannt, dass durch Histamin und Pepton produzierbare Shockzustände dem anaphylaktischen Shock überaus ähnlich sind. Über die Verschiedenheiten zwischen drei Shockformen sind recht zahlreiche Arbeiten in der Literatur niedergelegt worden, ohne jedoch zu allgemein gültigem Resultat gelangt zu sein.

Manwaring, ${ }^{2 / 8)}$ Dale u. Richards, ${ }^{3)}$ Dale u. Laidla $w^{4}$ u. a. haben den Histaminshock, Biedle u. Kraus, ${ }^{\text {, }}$ Pfeiffer u. Mita, ${ }^{6}$ ) Mautner u. Pick, ${ }^{7}$ Kritschewsky u. Friede ${ }^{9}$ u. a. den Pepton-

1) Tsuge, Tohoku Journ. Exp. Med.; 1938, 32, 531.

9) Ma nwaring, Ztschr. f. Immun.-Forsch., 1911, 8, 1.

3) Dale u. Richards, Journ. Physiol., 1918, 52, 110.

4) Dale u. Laidlaw, Ibid., 1919, 52, 355.

5) Biedle u. Kra us, Wien. klin. Wsehr., 1909, 363.

6) Pleiffer M. Mita, Ztschr. f. Immun.-Forsoh., 1910, 4, 410.

7) Ma utner u. Piek, Biochem. Ztschr., 1922, 127, 72.

8) Na nwaring, Journ. Immunol., 1923, 8, 211.

a) Kritschewsky u. Friede, Ztschr. f. Immun.-Forseh., 1925, 43, 1. 
shock-nach verschiedenen, bei jeder Shockform auftretenden Symptomen beurteilend-als beinahe identisch mit dem anaphylaktischen Shock angesprochen. In den letzten Jahren vertreten aber eine Reihe von Autoren den Stand punkt, dass, obzwar zwischen drei Shockformen teilweise Analogie besteht, gleichwohl sich eine Grenze zwischen ihnen ziehen lässt.

Um Beispiele dafür anzufïhren, haben $\mathrm{Manwaring,} \mathrm{Chil} \mathrm{coto,} \mathrm{Clark} \mathrm{u.}$ Monac ${ }^{10}$ den Beweis erbracht, dass, während beim Hund mit ausgeschalteter Leber der anaphylaktische und Peptonshock nie auftreten, bei ein und demselben Tier ein, wenn auch nicht typischer schwerer, doch immerhin Histamiashock erzeugt werden kann. Smith $\mathrm{h}^{1)}$ will an Kaninchen und Meersehweinchen nachgewiesen haben, dass durch die Histamininjektion die Körpertemperatursenkung und die Ungerinnbarkeit des Blutes, wie sie beim anaphylaktischen Shock beobachtet werden, vermisst werden. $\mathrm{Y}$ urin $\mathrm{o}^{12}$ und $\mathrm{Ball} \mathrm{y}^{13)}$ haben berichtet, dass beim anaphylaktischen Shock desKaninchens erheblicherKörpertemperatursturz sich findet, was beim Histaminshock hingegen nicht der Fall ist. L u m ière u. Malespin $\mathrm{e}^{14)}$ haben die Beobachtung gemacht, dass beim anaphylaktischen Shock des Meerschweinchens das Blut ungerinnbar ist, wohingegen beim Histaminshock nicht ungerinnbar ist.

$\mathrm{Zunz}$ u. La B arr $\mathrm{e}^{15)}$ sahen beim anaphylaktischen Shock des Meerschweinchens sowohl freie Zucker wie auch gebundene Zncker zunehmen; im Gegensatz hierzu hat La B a rre ${ }^{(6)}$ nachgewiesen, dass beim Histamin- und Peptonshock freie Zucker zunehmen, gebundene Zucker umgekehrt abnehmen. Oka moto ${ }^{\text {(t) }}$ hat an Kaninchen auch gleiche Tatsache festgestellt. Yoshid ${ }^{18)}$ hat an Meerschweinchen aus demVerhalten der Reaktionen auf verschiedenePharmaca deutliche Unterschiede zwischen dem anaphylaktischen und Hitamin- sowie Peptonshock konstatiert. Morio k $\mathrm{a}^{19)}$ gibt an, dass beim anaphylaktischen Shock des Kaninchens das Albumin abnimmt, das Gesamteiweiss und Globulin, insbesondere das letztere in erheblichem Masse zunimmt, während bein Histamin- und Peptonshock Gesamteiweiss, Albumin und Globulin gemeinschaftlich auffallend abnehmen.

Es haben Se eliger u. Gork $\mathrm{e}^{20)}$ im anaphylaktischen und Peptonshock des Kaninchens, $\mathrm{M}$ a e d $\mathbf{a}^{21)}$ im ana phylalstischen und Histaminshock des Meerschwein-

10) Manwaring, Chilcote, Clark u. Monaco, Proc. Soc. Exp. Biol. \& Med., 1922-23, 20, 182.

11) Smith, Journ. Immunol., 1920, 5, 239.

12) Y urino, Fukuoka Fkadaigaku Zasshi, 1925, 18, 386.

13) Bally, Journ. Immunol, 1929, 17, 191 u. 223.

14) Lumière a. Males pine, C. Y. Soc. Biol. Paris, 1930, 104, 1010.

15) Zunz u. La Barre, Ibid., 1924, 91, 132.

16) La Barre, Ibid., 1927, 97, 725.

17) Okamoto, Kyoto Furitsu Ikadaigaku Zasshi, 1932, 6, 937.

18) Yoshida, Keio.Igaku, 1930, 10, 459.

19) Morioka, Kyoto Furitsu Ilsadaigaku Zasshi, 1933, 7, 703.

20) Seeliger u. Gorke, Ztschr. exp. Med., 1921, 24, 322.

21) Ma ed a, Nippon Naibumpigakkai Zasshi, 1929-30, 5, 1974. 
chens korpuskuläre Elemente des Blutes gegeneinander vergleichend analysiert und gefunden, dass zwischen beiden Shockformen jede für sich grosse Unterschiede bestehen. D juri ci $\mathrm{c}^{22)}$ gibt an, dass bei Hunden die Glykose auf das Auftreten anaphylaktischen Shocks hemmend wirkt, während die Blutd rucksenkung durch Histamin durch Glykose nicht beeinflusst wird. Nach J u n g $^{23)}$ gewinnt das Blut im anaphylaktischen Shock des Hundes eine übertragbare stark blutdrucksenkende Eigensehaft, wohingegen es beim Peptonshock kein oder eine nichtnenmenswerte hypotensive Eigenschaft besitzt. Puccinelli u. Agostino $0^{24}$ haben an weissen Ratten nachgewiesen, dass der Blutdruck im Histaminshock rou Anfang an sinkt, beim Peptonshock zuerst sinkt, dann steigt.

Indessen ist von allen Autoren übereinstimmend anerkannt worden, dass der Histamin- und Peptonshock ebenso wie der anaphylaktische Shock in inniger Beziehung zur Leber steht. Nach Meinung von Ma nw arin $g^{2)}$ soll sich beim anaphylaktischen Shock ein histaminartigrer Stoff (Anaphylatoxin) aus der Leber explosionsartig ausschwemmen und so Shocksymptome hervorrufen. Mautner u. Pick, Eppinger u. Leuchtenberger ${ }^{25}$ haben beim Histaminshock, Mautner u. Pick, ${ }^{7}$ Manwaring;, ${ }^{8)}$ Weil, ${ }^{26}$ ) Teraoka ${ }^{27}$ beim Peptonshock histologische Untersuchung der Leber ausgefüht und gefunden, dass die Leberparenchymzellen degenerative Veränderungen aufwiesen, indem sie ödematös angeschwollen und getribt waren.

Nach alledem wäre es leicht denkbar, dass beim Histamin- und Peptonshock der intermediäre Kohlehydratstoffwechsel in eben ähnlicher Weise, wie im anaphylaktischen Shock, markante Veränderungen erfahren muss; gleichwohl lauten diesbezügliche Angaben von verschiedenen Autoren ziemlich widersprechend.

Was das Verhalten des Blutzuckers im Histaminshock anbelangt, haben Chambers u. Thompson ${ }^{28}$ beim Hund, La Barre ${ }^{29}$ beim Meerschweinchen, Kinoshita, $\left.{ }^{30)} \mathrm{Okamoto},{ }^{17)} \mathrm{Matsueda},{ }^{31}\right) \mathrm{Mae}$ $\mathrm{da}^{32}{ }^{32}$ beim Kaninchen die Hyperglykämie nachgewiesen, Sch en $\mathbf{k}^{33}$ ) will aber keine Veränderung des Blutzuckers gesehen haben; A o ki $i^{34}$

22) D juricic, C. r. Soc. Biol. Paris, 1933, 113, 432.

23) Jung, Ibid., 1933, 114, 56.

24) Puceinelliu. Agost in o, Pathologica (Genova), 1933, 25, 153.

25) Eppinger u. Leuchtenberger, Ztschr. exp. Med., 1932, 85, 581.

26) W e i 1, Journ. Immunol, 1917, 2, 525̃.

27) Tera oka, Tokyo Igakkai Zasshi 1936, 50, 101.

28) Chambers u. Thompso n, Journ. Infec. Disease, 1925, 37, 229.

29) L a Barre, C. r. Soc. Biol. Paris, 1926, 94, 779.

30) Kinos hita, Igaku. Kenkyu, 1928, 2, 899.

31) Matsued a, Okayama Igakkai Zasshi, 1932, 44, 859.

32) Maeda, Nagasaki Igakkai Zasshi, 1933, 11, 1458.

33) S ehenk, Arch. f. exp. Path. u. Pharm., 1921, 89, 332.

34) Aoki, Nippon Byorigakkai Kaishi, 1930, 19, 317. 
und $\mathrm{Ohmi} \mathrm{i}^{35}$ ) berichteten über das Auftreten der Hypoglykämie. Osato ${ }^{36)}$ Achard u. Feuillie ${ }^{37)}$ und M. Watanab e ${ }^{38)}$ habenbeim Hund im Peptonshock die Hyperglykämie konstatiert ; entgegen diesen Angaben wollen Mc Guigan u. Ross ${ }^{39}$ ) Mizuhar ${ }^{40)}$ die Hypog:lykämie bemerkt haben. Beim Kaninchen haben eine ganze Reihe von Autoren (Kuriyama, ${ }^{41)}$ Mizuhara, ${ }^{40)}$ Kato, ${ }^{42)}$ Nakanishi, ${ }^{43)}$ Okamoto, ${ }^{17)}$ Kaiwa, ${ }^{44)}$ H. Watanabe ${ }^{45)}$ u.a.m.) die Hyperglykämie auftreten gesehén.

Über die Blutmilchsäure sind literarische Angaben äusserst spärlich, es liegen nämlich nur Untersuchungen von Chambers u. Th o m $\mathrm{p}$ so $n$ vor, in denen sie im Histaminshock das Auftreten von Hyperlactacidämie nachwiesen. Histamin und Pepton sind schon früher durch Untersuchungen von Heidenhain, ${ }^{46)}$ Starling, ${ }^{47}$ Dale $u$. Laid la $w^{4}$ u. a. als Lymphagoga bekannt, beim durch sie ausgelösten Shock soll die Lymphbildung sich sehr lebhaft vollziehen, wobei zugleich die Bluteindickung eintritt. Im Histaminshock haben $\mathrm{D}$ ale $\mathrm{u}$. Laidlaw, Mautner. u. Pick, Dale, ${ }^{48)}$ Sato, ${ }^{49)}$ Maeda, ${ }^{50)}$ Dérer u. Steffanutti, ${ }^{51)}$ Eppinger u. Lenchtenberger, ${ }^{25)}$ Puddu u. Vittorio die Bluteindickung konstatiert; Adlersberg u. Paul ${ }^{53}$ ) haben aus der Wasseravidität der Haut auf Störungen des Wasserstoffwechsels geschlossen. Im Peptonshock haben Heide $\mathrm{nh}$ hin, ${ }^{46)} \mathrm{Star}^{-}$ ling, ${ }^{47}$ Henderson u. Underhill, ${ }^{54)}$ Mautner u. Pick, ${ }^{7}$ Manwaring, ${ }^{8)}$ Sato, $\left.{ }^{49}\right)$ Teraoka ${ }^{27}$ ) die Bluteindickung nachgewiesen.

Wie aus obigen Anführungen klar hervorgeht, steht es nunmehr ausser allem Zweifel, dass beim Histamin- und Peptonshock der intermediäre Kohlehydrat- und Wasserstoffwechsel mannigfache Verände-

\footnotetext{
35) O h mi, Tohoku Journ. Exp. Med., 1933, 21, 314.

36) Os a to, Ibid., 1921, 2, 487.

37) Achard u. Feuillié, C. x. Soc. Biol. Paris, 1922, 86, 760.

38) M. W atanabe, Tohokn Journ. Exp. Med., 1927, 9, 412.

39) Me Guigan u. Ross, Journ. Biol. Chem., 1917, 29, 129.

40) Mizuhara, Tokjo Igakkai Zasshi, 1920, 34, 707.

41) Kuriy a m a, Journ. Biol. Chem, 1917, 29, 127.

42) Ka to, Iji Shimbun, 1924, Nr. 1144, 817.

43) Nakanishi, Keio Igaku, 1929, 9, 1279

44) Ka iwa, Tohoku Journ. Exp. Med., 1933, 20, 365.

4b) H. W atanabe, Ibid., 1935, 27, 416.

46) Heiden ha in, Arch. f. ges. Physiol., 1891, 49, 209.

47) Starling, Journ. Physiol, 1894, 16, 224.

48) Da I e, Brit. Journ. Exp. Path., 1920, 1, 103.

49) Sato, Shakai Igaku Zasshi, 1928, 1263.

50) Ma eda, Nippon Naibumpigakkai Zasshi, 1930, 5, 2178.

51) Dérer u. Steffanutti, Biochem. Ztschr., 1930, 223, 408.

52) Puddu u. Vittorio, Boll. Soe. ital. Biol. sper., 1934, 9, 151.

53) Adlersberg u. Pa ul, Ztsehr. exp. Med., 1934, 92, 293.

54) Henders on u. Underhill, Amer. Journ. Physiol., 1911, 28, 275.
} 
rungen exfahren, die mit der mit dem Shock einheigehenden Funktionsstörung der Leber in überaus engem Zusammenhang stehen. In Anbetracht dieser Sachlage erscheint mir äusserst interessant, Untersuchungen darüber anzustellen, ob und inwieweit Stoffumsätze in der Leber in situ bei solchen Tieren, die in diese Shockzustände versetzt sind, beeinflusst werden, und welche Unterschiede zwischen dem anaphylaktischen Shock und diesen zwei Shockformen bestehen.

In vorliegenden Versuchen habe ich unter ganz demselben Verfahren, welches in einschlägige Studien über den anaphylaktischen Shock herangezogen wurde, an Kaninchen in zweierlei Shockzüständen (im Histamin- und Peptonshock) den Blutmilchsäure-, Blutzucker-, Hämoglobin- und Serumeiweissgehalt im arterriellen, Pfortader- und Lebervenenblut bestimmt ind so gewonnene Werte zeitlich vergleichend analysiert.

Die Ergebnisse des Kontrollversuchs sind bereits in der I. Mitteilung.1) geschildert worden. Nach diesen Ergebnissen erweist sich der Milchsäuregehalt normalerweise im arteriellen Blut als maximal, im Pfortaderblutals mittelmässig und im Lebervenenblutals minimal. De: Zuckerspiegel des Lebervenenblutes bietet hingegen den höchsten Wertt dar, demselben folgt das Arterienblut, das Pfortaderblut weist den Mindestwert auf. Das Hämoglobin zeigt in drei Blutarten fast gleiche Werte, die sich voneinander kaum unterscheiden lassen. Der Serumeiweissgehalt ist je nach den Individuen verschieden gross, so dass man sich kein einheitliehes Bild darüber verschaffen kann, im grossen ganzen jedoch ist der Serumeiweissgehalt des Lebervenenblutes am niedrigsten.

\section{Histaminshock.}

Zwecks Shockauslösung wurde gesunden, ungefähr $2 \mathrm{~kg}$ schweren Kaninchen 0,3-0,5 ccm einer 0,1\% igen Histaminlösung (Merck) pro $\mathrm{kg}$ Körpergewicht so schnell als möglich intravenös injiziert. Der Shock trat ungefähr 10 Sek.-2 Min. nach Beendigung der Injektion auf, die Symptome waren ganz dieselben, wie die im anaphylaktischen Shock.

Blutmilchsäure: An 7 Versuchen gewonnene Ergebnisse sind in Tab. 1 aufgezeichnet. Fig. 1 stellt ein Versuchsbeispiel dar. Die Reihenfolge des Milchsäuregehaltes in drei Blutarten vor der Injektion verhielt sich natürlich ganz gleich wie im Kontrollversuch. Unmittelbar nach Shockausbruch nahm die Blutmilchsäure im arteriellen Blut 
in Versuch 2 und $5 \mathrm{zu}$, in übrigen 5 Versuchen umgekehrt ab, besonders erheblich war diese Abnahme in Versuch 1,6 und 7. Im Pfortaderblut waren in sämtlichen Versuchen deutliche Zunahmen anzutreffen. In Lebervenenblut wurde, ausgenommen den Versuch 7 mit Abnahme, ebenso auch erhebliche Zunahme ermittelt; nach dem durchschnittlichen Prozentsatz berechnet, war die prozentische Zunahme im Lebervenenblut am höchsten.

Im Ablauf von 15 Minuten traten im arteriellen und Pfortaderblut Zunahmen in sämtlichen Versuchen auf, im Lebervenenblut erfuhr die Blutmilchsäure, mit Ausnahme des Versuchs 6, in allen übrigen Versuchen beträchtliche Zunahme, welche in den meisten Versuchen dieselbe in anderen zwei Blutarten ibertraf. In 3 Versuchen, bei denen wegen

\section{Tabelle 1 .}

Veränderungen des Blutmilchsäurespiegels in der Leber beim Histaminshock.

\begin{tabular}{|c|c|c|c|c|c|c|c|c|c|}
\hline \multirow{2}{*}{$\begin{array}{l}0 \\
3 \\
0 \\
0 \\
0\end{array}$} & \multirow{2}{*}{ 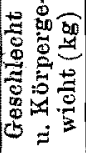 } & \multirow{2}{*}{$\begin{array}{l}\text { Zeit der } \\
\text { Blutent } \\
\text { nahme } \\
\text { (Min.) }\end{array}$} & \multicolumn{2}{|c|}{ A. carotis } & \multicolumn{2}{|c|}{ V. portae } & \multicolumn{2}{|c|}{$\nabla$. hepatica } & \multirow{2}{*}{ Bemerkungen } \\
\hline & & & $\mathrm{mg} / \mathrm{dl}$ & $\begin{array}{c}\text { Diff. in } \\
\%\end{array}$ & $\mathrm{mg} / \mathrm{dl}$ & $\underset{\%}{\text { Diff. in }}$ & $\mathrm{mg} / \mathrm{dl}$ & 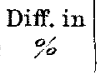 & \\
\hline 1 & $\stackrel{8}{1,90}$ & $\begin{array}{l}\text { Vor d. Inj. } \\
\text { Nach } 3 \text { Min. } \\
, 15,\end{array}$ & $\begin{array}{l}39,21 \\
36,00 \\
44,36\end{array}$ & $\begin{array}{r}-8,2 \\
+\quad 13, y\end{array}$ & $\left|\begin{array}{l}41,79 \\
52,07\end{array}\right|$ & $\begin{array}{l}+10,2 \\
+\quad 37,3\end{array}$ & $\left|\begin{array}{l}32,14 \\
42,43 \\
57,86\end{array}\right|$ & $\begin{array}{r} \\
+\quad 32,0 \\
+\quad 80,0\end{array}$ & $\begin{array}{l}50 \text { Sekunden nach } \\
\text { Injektion Krämpfe, } \\
\text { Dauer einige Min. } 29 \\
\text { Min. nach Injektion } \\
\text { Herzstillstand. } \\
\text { Shock (H) }\end{array}$ \\
\hline 2 & $\stackrel{?}{1,98}$ & $\begin{array}{l}\text { Vor d. Inj. } \\
\text { Nach } 3 \text { Mlin, } \\
\quad 15,\end{array}$ & $\left|\begin{array}{l}23,79 \\
25,07 \\
39,21\end{array}\right|$ & $\begin{array}{r}+ \\
+\quad 5,4 \\
+\quad 64,8\end{array}$ & $\mid \begin{array}{r}23,14 \\
38,57 \\
42,43\end{array}$ & $\begin{array}{r} \\
+\quad 66,7 \\
+\quad 83,4\end{array}$ & $\begin{array}{l}19,29 \\
25,71 \\
43,07\end{array}$ & $\begin{array}{r} \\
+\quad 33,3 \\
+123,3\end{array}$ & $\begin{array}{l}\text { Cyanose, Unruhe, be- } \\
\text { schleunigte Atmung. } \\
\text { Kein Krampt. Shork } \\
(+)\end{array}$ \\
\hline 3 & $\frac{9}{2,51}$ & $\begin{array}{l}\text { Vord. Inj. } \\
\text { Nach } 3 \text { Min. }\end{array}$ & $\begin{array}{l}\mathbf{2 9} 9 \mathbf{5 7} \\
\mathbf{2 8 , 2 9}\end{array}$ & 4,3 & $\begin{array}{r}28,93 \\
32,79\end{array}$ & $+13,3$ & $\left|\begin{array}{l}22,50 \\
34,47\end{array}\right|$ & $+53,2$ & $\begin{array}{l}\text { Gleich nach Injektion } \\
\text { Krämple. } 71 \text { Min. } \\
\text { nach Injektion Herz- } \\
\text { stillstand. Shock }(H+)\end{array}$ \\
\hline 4 & $\begin{array}{l}\text { 울. } \\
2,31\end{array}$ & $\begin{array}{l}\text { Vor d. Inj. } \\
\text { Nach } 3 \text { Min. }\end{array}$ & \begin{tabular}{|}
33,43 \\
32,79
\end{tabular} & $\begin{array}{r}-\quad 1,9 \\
-\end{array}$ & $\begin{array}{l}28,93 \\
45,00\end{array}$ & $+55,5$ & $\mid \begin{array}{l}25,07 \\
47,57\end{array}$ & $+89,7$ & $\begin{array}{l}\text { I Min. nach Injektion } \\
\text { Krämpfe. } 5 \text { Min. } \\
\text { nach Injektion ge- } \\
\text { storben. Shock (Hi) } \\
\end{array}$ \\
\hline 5 & 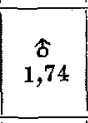 & \begin{tabular}{|} 
Vor d. Inj. \\
Nach 5 Min. \\
$\Rightarrow 15 \quad$, \\
300,
\end{tabular} & $\begin{array}{l}23,79 \\
30,21 \\
38,57 \\
52,71 \\
\end{array}$ & $\begin{array}{r}+27,0 \\
+\quad 62,1 \\
+121,6 \\
\end{array}$ & $\begin{array}{l}22,50 \\
36,00 \\
41,79 \\
55,29\end{array}$ & $\begin{array}{r}+60,0 \\
+\quad 85,7 \\
+145,7 \\
\end{array}$ & \begin{tabular}{|l|}
18,64 \\
30,21 \\
45,64 \\
64,29
\end{tabular} & $\begin{array}{r}+62,1 \\
+144,8 \\
+244,9 \\
\end{array}$ & $\begin{array}{l}\text { Unruhig, Atmung be- } \\
\text { schleunigt. Kein } \\
\text { Krampt. Shoek }(+)\end{array}$ \\
\hline 6 & $\stackrel{\text { 울 }}{1,95}$ & $\begin{array}{c}\text { Vor d. Inj. } \\
\text { Naoh 5-Min. } \\
" 15, \\
" 30,\end{array}$ & $\begin{array}{l}40,50 \\
37,29 \\
41,14 \\
45,00\end{array}$ & $\begin{array}{r}-\quad 7,9 \\
+\quad 1,6 \\
+\quad 11,1 \\
\end{array}$ & $\begin{array}{l}31,50 \\
34,71 \\
41,79 \\
48,86\end{array}$ & $\begin{array}{r}+ \\
+\quad 10,2 \\
+\quad 32,7 \\
+\quad 55,1 \\
\end{array}$ & \begin{tabular}{|l|}
27,64 \\
37,29 \\
34,71 \\
44,86 \\
\end{tabular} & $\begin{array}{r}+34,9 \\
+\quad 25,6 \\
+\quad 60,5 \\
\end{array}$ & $\begin{array}{l}\text { Unruhig, besehleu- } \\
\text { nigte Atmung } 30 \text { Se- } \\
\text { kunden nach Injek- } \\
\text { tion. Shock }(+)\end{array}$ \\
\hline 7 & $\stackrel{8}{1,52}$ & $\begin{array}{l}\text { Vor d. Inj. } \\
\text { Nach } 5 \text { Min. } \\
\text { " } 15 \text { " } \\
\text { "30 },\end{array}$ & $\left|\begin{array}{l}37,29 \\
32,14 \\
47,29 \\
55,29\end{array}\right|$ & $\begin{array}{r}-13,8 \\
+\quad 26,8 \\
+\quad 48,3\end{array}$ & $\begin{array}{l}30,21 \\
36,64 \\
41,79 \\
54,00\end{array}$ & $\begin{array}{r}+\quad 21,3 \\
+\quad 38,3 \\
+\quad 78,7\end{array}$ & $\left|\begin{array}{l}28,93 \\
25,71 \\
37,93 \\
63,00\end{array}\right|$ & $\left|\begin{array}{r}11,1 \\
+\quad 31,1 \\
+117,8\end{array}\right|$ & $\begin{array}{l}\text { Atmung beschleunigt. } \\
\text { Cyanose leichten } \\
\text { Grades. Shook }( \pm)\end{array}$ \\
\hline
\end{tabular}


Fïg. 1. Veränderungen des Milchsäuregehaltes des arteriellen, des Pfortader- und des Lebervenenblutes beim Histaminshock (Versuch 1).

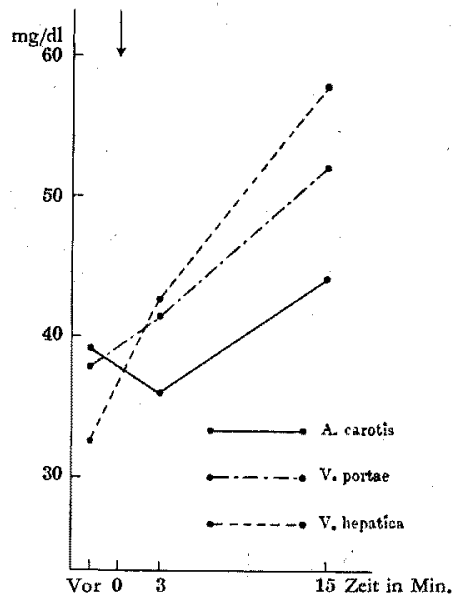

, geringfügiger Shocksymptome eine 30 Minuten währende Beobachtung: möglich war, erhöhte sich die Blutmilchsäure in drei Blutarten weiter fortgesetrt; diese Erhöhung trat besonders intensiv im Lebervenenblut und zwar am ausgeprägsten in Versuch 5 u. 7 auf.

Wie aus obigen Ergebnissen ersichtlich ist, erfährt der Milchsäuregehalt unmittelbar nach Shockausbruch, abgesehen von initialer Abnahme im arteriellen Blut, in anderen zwei Blutarten erhebliche Zunahmen, und nachher nimmt die Milchsäure in drei Blutarten mit dem Zeitablauf immer mehr zu. Die prozentische Zunahme ist im Lebervenenblut am grössten, im Pfortaderblut weniger gross und im Arterienblut am kleinsten. Aus derartigem Verhalten der Blutmilchsäure kann zwangsläufig geschlossen werden, dass in der Leber die lebhafte Milchsäurebildung vonstatten geht.

Blutzucker: An 6 Kaninchen erhaltene Ergebnisse sind in Tab. 2 und Fig. 2 neben den Hämoglobin- und Ejweisswerten zusammengestellt.

Die Reihenfolge des Zuckerwertes in drei Blutarten vor der Injektion war ganz dieselbe wie die im Kontrollversuch.

Unmittelbar nach Shockausbruch wurde die Erhöhung des Zuckerspiegels in drei Blutarten gemeinschaftlich gefunden. In Versuch 4 u. 5 war die Erhöhung im arteriellen Blut am grössten, während in Versuch 3 und 4 die Erhöhung im Lebervenenblut am hervorragendsten war. Der Zuckerspiegel verhält sich hierand ers als im anaphylaktischen Shock, wo der: Zuckerspiegel im arteriellen und Pfortaderblut fast unverändert bleibt, nur im Lebervenenblut allein erheblich erhöht war, indem er beim vorliegenden Shock auch im arteriellen und Pfortaderblut deutliche Erhöhung aufweist.

Im Ablauf von 15 Minuten zeigte der Zuckerspiegel recht verschiedentliche Verhältnisse. Der Zuckerspiegel im Lebervenenblut nämlich wax in Versuch 5 erhöht und, ausgenommen den Versuch 6 


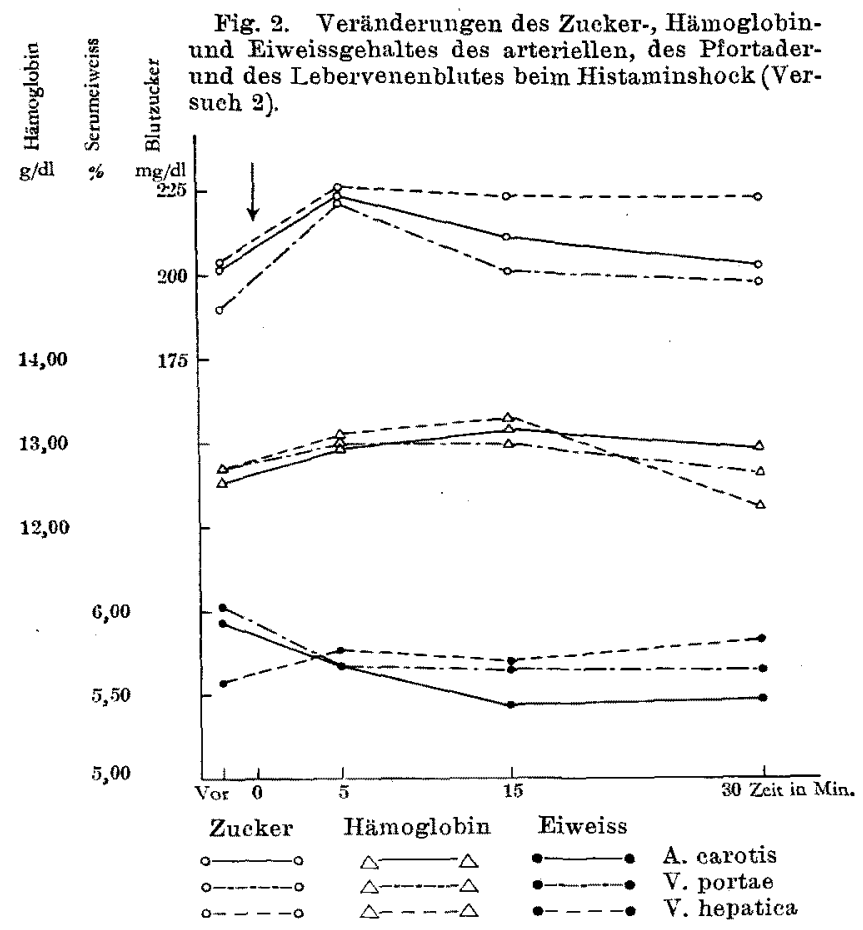

mit konstantem Wert, in allen übrigen Versuchen erniedrigt, ohne je auf den Anfangswert zurickgekommen zu sein. Im arteriellen und Pfortaderblut wechselten Zunahme und Abnahme miteinander zur Hälfte $a b$, indem der Zuckerspiegel in Versuch 1, 3 und 5 noch weitere Erhöhung, in Versuch 2, 4 und 6 erniedrigte Werte aufwies, die dennoch iuber Ursprungswerte liegen. Nach 30 Minuten zeigte der Zuckerspiegel in drei genannten Blutarten nur in Versuch 2 die Tendenz zu geringer Abnahme, während er in anderen 3 Versuchen in drei Blutarten miteinander fast parallel erhöht war.

Im Histaminshock tritt nämlich die Erhöhung des Zuckerspiegels in drei Blutarten deutlich auf, wobei sich der Zuckerspiegel des Lebervenenblutes meistenfalls gegenüber demselben des Pfortaderblutes stärker erhöht. Dies dürfte höchstwahrscheinlich von spärlichem Zuckerschwund in der Leber hergerührt haben.

Häm oglobin: Unmittelbar nach Shockausbruch traten Zunahmen in drei Blutarten auf und zwar im Lebervenenblut um ein Geringes 
Tabelle

Veränderungen des Blutzucker-, Hämoglobin- und

\begin{tabular}{|c|c|c|c|c|c|c|c|c|c|c|c|c|}
\hline \multirow{3}{*}{$\begin{array}{l}\text { Ver- } \\
\text { such }\end{array}$} & \multirow{3}{*}{$\begin{array}{c}\text { Geseh- } \\
\text { lecht } \\
\text { n. } \\
\text { Körper- } \\
\text { gewieht } \\
\text { (kg) }\end{array}$} & \multirow{3}{*}{$\begin{array}{c}\text { Zeit der } \\
\text { Blutent- } \\
\text { nahme } \\
\text { (Min.) }\end{array}$} & \multicolumn{6}{|c|}{ A. earotis } & \multicolumn{4}{|c|}{ V. poutare } \\
\hline & & & \multicolumn{2}{|c|}{ Zucker } & \multicolumn{2}{|c|}{$\mathrm{Hb}$} & \multicolumn{2}{|c|}{$\begin{array}{l}\text { Serumei- } \\
\text { weiss }\end{array}$} & \multicolumn{2}{|c|}{ Zucker } & \multicolumn{2}{|c|}{$\mathrm{Hb}$} \\
\hline & & & $\begin{array}{c}\mathrm{mg} / \\
\mathrm{dl}\end{array}$ & $\begin{array}{l}\text { Diff. } \\
\text { in } \%\end{array}$ & $g / d 1$ & $\begin{array}{l}\text { Diff:. } \\
\text { in } \%\end{array}$ & $\%$ & Diff. & $\begin{array}{c}\mathrm{mg} / \\
\mathrm{dI} /\end{array}$ & $\begin{array}{l}\text { Diff. } \\
\text { in } \%\end{array}$ & $\mathrm{~g} / \mathrm{dl}$ & $\begin{array}{l}\text { Diff: } \\
\text { in } \%\end{array}$ \\
\hline 1 & 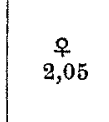 & 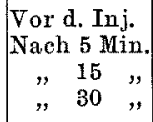 & $\begin{array}{l}191 \\
217 \\
232 \\
251\end{array}$ & $\begin{array}{l}+13,6 \\
+21,5 \\
+31,4\end{array}$ & $\begin{array}{l}12,20 \\
12,24 \\
11,94 \\
11,60\end{array}$ & $\begin{array}{r}+0,3 \\
-2,1 \\
-4,9\end{array}$ & $\begin{array}{l}5,68 \\
5,62 \\
5,56 \\
5,34\end{array}$ & $\begin{array}{r}-1,0 \\
-2,1 \\
-6,0\end{array}$ & $\begin{array}{l}195 \\
218 \\
226 \\
245\end{array}$ & $\begin{array}{l}+11,8 \\
+15,9 \\
+25,6\end{array}$ & $\begin{array}{l}12,20 \\
12,24 \\
11,96 \\
11,66\end{array}$ & $\begin{array}{r}+0,3 \\
-2,0 \\
-4,4\end{array}$ \\
\hline 2 & $\begin{array}{c}\hat{\delta} \\
1,85\end{array}$ & 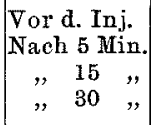 & $\begin{array}{l}202 \\
222 \\
211 \\
206\end{array}$ & $\begin{array}{l}+9,9 \\
+4,4 \\
+2,0\end{array}$ & $\begin{array}{l}12,57 \\
12,95 \\
13,13 \\
12,94\end{array}$ & $\begin{array}{r}+3,0 \\
+4,4 \\
+2,9\end{array}$ & $\begin{array}{l}5,88 \\
5,67 \\
5,47 \\
5,49\end{array}$ & $\mid \begin{array}{r}-3,6 \\
-7,0 \\
-6,6\end{array}$ & $\begin{array}{l}193 \\
221 \\
202 \\
199\end{array}$ & $\begin{array}{r}+14,5 \\
+4,7 \\
+3,1\end{array}$ & $\begin{array}{l}12,61 \\
12,98 \\
12,98 \\
12,67\end{array}$ & $\begin{array}{r}+2,9 \\
+2,9 \\
+0,5\end{array}$ \\
\hline 3 & 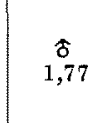 & $\begin{array}{l}\text { Vor d. Inj. } \\
\text { Naeh } 5 \text { Min. } \\
" 15 \\
" 15\end{array}$ & $\begin{array}{l}181 \\
184 \\
226 \\
228\end{array}$ & $\begin{array}{r}+1,6 \\
+24,9 \\
+26,0\end{array}$ & $\begin{array}{l}15,92 \\
16,32 \\
15,88 \\
15,08\end{array}$ & $\begin{array}{r}+2,5 \\
-0,2 \\
-5,3\end{array}$ & $\begin{array}{l}6,03 \\
5,81 \\
5,79 \\
5,68\end{array}$ & $\begin{array}{l}-3,6 \\
-4,0 \\
-5,8\end{array}$ & $\begin{array}{l}163 \\
170 \\
222 \\
228\end{array}$ & $\begin{array}{r}+4,3 \\
+36,2 \\
+39,9\end{array}$ & $\begin{array}{l}15,92 \\
16,32 \\
15,88 \\
15,08\end{array}$ & $\begin{array}{r}+2,5 \\
-0,9 \\
-5,3\end{array}$ \\
\hline 4 & $\begin{array}{c}\hat{\delta} \\
1,80\end{array}$ & $\begin{array}{l}\text { Vor d. Tnj. } \\
\text { Nach } 3 \text { Min. } \\
\Rightarrow 15,\end{array}$ & $\begin{array}{l}193 \\
232 \\
206\end{array}$ & $\begin{array}{r}+20,2 \\
+6,7\end{array}$ & $\left|\begin{array}{l}14,70 \\
14,94 \\
13,36\end{array}\right|$ & $\begin{array}{r}+1,6 \\
-9,1\end{array}$ & $\begin{array}{l}6,03 \\
5,67 \\
5,94\end{array}$ & $\begin{array}{r}-6,0 \\
-1,5\end{array}$ & $\begin{array}{l}186 \\
211 \\
193\end{array}$ & $\begin{array}{r}+13,4 \\
+3,8\end{array}$ & $\begin{array}{r}14,70 \\
14,80 \\
13,64\end{array}$ & $\begin{array}{l}+0,7 \\
-7,2\end{array}$ \\
\hline 5 & $\begin{array}{c}\text { 古 } \\
1,92\end{array}$ & $\begin{array}{l}\text { Vor d. } \operatorname{Tn} \mathrm{j} . \\
\text { Nach } 5 \text { Min. } \\
\text { " } 15,\end{array}$ & $\begin{array}{l}175 \\
215 \\
251\end{array}$ & $\begin{array}{l}+22,8 \\
+43,4\end{array}$ & $\begin{array}{l}15,26 \\
15,98 \\
15,98\end{array}$ & $\begin{array}{l}+4,7 \\
+4,7\end{array}$ & $\begin{array}{l}6,19 \\
5,90 \\
6,34\end{array}$ & $\begin{array}{r}-4,7 \\
+2,4\end{array}$ & $\begin{array}{l}172 \\
188 \\
206\end{array}$ & $\begin{array}{r}+9,3 \\
+19,8\end{array}$ & $\begin{array}{r}15,26 \\
15,98 \\
15,64\end{array}$ & $\begin{array}{l}+4,7 \\
+2,5\end{array}$ \\
\hline 6 & $\stackrel{\delta}{\delta}$ & $\begin{array}{l}\text { Vor d. Inj. } \\
\text { Nach } 3 \text { Min. } \\
\begin{array}{ccc} & 15 & \\
" & 30 & \end{array}\end{array}$ & $\begin{array}{l}166 \\
195 \\
175 \\
191\end{array}$ & $\begin{array}{r}+17,5 \\
+5,4 \\
+15,1\end{array}$ & $\begin{array}{l}15,79 \\
16,10 \\
15,20 \\
15,10\end{array}$ & $\begin{array}{r} \\
+2,0 \\
-3,7 \\
-4,4\end{array}$ & $\begin{array}{l}6,08 \\
6,08 \\
6,03 \\
5,93\end{array}$ & $\begin{array}{l} \pm 0 \\
-0,8 \\
-2,5\end{array}$ & $\begin{array}{l}164 \\
177 \\
172 \\
174\end{array}$ & $\begin{array}{l}+7,9 \\
+49 \\
+6,1\end{array}$ & $\begin{array}{l}15,79 \\
16,12 \\
15,20 \\
15,10\end{array}$ & $\begin{array}{r}+2,1 \\
-3,7 \\
-4,4\end{array}$ \\
\hline
\end{tabular}

stärker als in anderen zwei Blutarten. Bis zum Ablauf von 15 Minuten erfuhr der Hämoglobingehalt, ausgenommen den Versuch 2, wo noch weitere Zunahme im Lebervenenblut gefunden wurde, in den meisten Versuchen eine Erniedrigung, deren Grad aber gegenüber der Kontrolle geringer war. Nach 30 Minuten trat in sämtlichen Versuchen eine Erniedrigung auf, die beinahe gleichgradig war wie bei der Kontrolle.

Hierbei habe ich mich nämlich davon überzeugt, dass der Hämoglobingehalt in drei Blutarten unmittelbar nach Shockausbruch nach vorangehender Zunahme allmählich immer mehr abnimmt, und dass trotzdem noch bis zum Ablauf von Viertelstunde eine mehr oder weniger Bluteindickung besteht, welche im Lebervenenblut ein wenig stärkè als in anderen zwei Blutarten ist. 
Kohlehydrat- u. Wasserstoffwechsel in Leber beim Histamin- n. Peptonshock 567.

2.

Eiweissgehaltes in der Leber beim Histaminshock.

\begin{tabular}{|c|c|c|c|c|c|c|c|c|}
\hline \multirow{2}{*}{\multicolumn{2}{|c|}{$\begin{array}{l}\text { Serumei- } \\
\text { weiss }\end{array}$}} & \multicolumn{6}{|c|}{ V. hepatica } & \multirow{3}{*}{ Bemerkungen } \\
\hline & & \multicolumn{2}{|c|}{ Zucker } & \multicolumn{2}{|c|}{$\mathbf{H b}$} & \multicolumn{2}{|c|}{$\begin{array}{l}\text { Serumei- } \\
\text { weiss } \%\end{array}$} & \\
\hline$\because$ & $\begin{array}{l}\text { Diff: } \\
\text { in } \%\end{array}$ & $\mathrm{dig} /$ & $\begin{array}{l}\text { Diff. } \\
\text { in } \%\end{array}$ & $\mathrm{~g} / \mathrm{dl}$ & $\begin{array}{l}\text { Diff. } \\
\text { in } \%\end{array}$ & $\%$ & $\begin{array}{l}\text { Diff. } \\
\text { in } \%\end{array}$ & \\
\hline $\begin{array}{l}5,70 \\
5,88 \\
5,56 \\
5,43\end{array}$ & $\begin{array}{l}+3,1 \\
-\mathbf{2}, 4 \\
-4,7 \\
\end{array}$ & $\begin{array}{l}222 \\
247 \\
236 \\
278\end{array}$ & $\begin{array}{r} \\
+11,3 \\
+6,3 \\
+25,2\end{array}$ & $\begin{array}{l}12,20 \\
12,50 \\
12,02 \\
11,36\end{array}$ & $\begin{array}{r}+2,4 \\
-1,5 \\
-6,9 \\
\end{array}$ & $\begin{array}{l}5,54 \\
5,99 \\
5,38 \\
5,27\end{array}$ & $\begin{array}{r}+8,1 \\
-2,9 \\
-4,9 \\
\end{array}$ & $\begin{array}{l}11_{2} \text { Min. naeh Injektion Atmung be- } \\
\text { schleunigt. Cyanose. Kein Krampf. } \\
\text { Shock }(+)\end{array}$ \\
\hline $\begin{array}{l}6,01 \\
5,67 \\
5,65 \\
5,67\end{array}$ & $\begin{array}{l}-5,6 \\
-6,0 \\
-5,6\end{array}$ & $\begin{array}{l}206 \\
226 \\
224 \\
223\end{array}$ & $\begin{array}{r}+9,7 \\
+8,7 \\
+8,2\end{array}$ & $\begin{array}{l}12,61 \\
13,08 \\
13,29 \\
12,24\end{array}$ & $\begin{array}{r}+3,7 \\
+5,4 \\
-2,9\end{array}$ & $\begin{array}{l}5,58 \\
5,70 \\
5,67 \\
5,77\end{array}$ & $\begin{array}{r}+2,1 \\
+1,6 \\
+3,4\end{array}$ & $\begin{array}{l}\text { 2 Min. nach Injektion unruhig, At- } \\
\text { mung beschlemigt. Kein Krampt. } \\
\text { Shoek }(+)\end{array}$ \\
\hline $\begin{array}{l}5,72 \\
5,60 \\
5,86 \\
5,79\end{array}$ & $\begin{array}{r}-2,1 \\
+2,4 \\
+1,2\end{array}$ & $\begin{array}{l}200 \\
243 \\
240 \\
255\end{array}$ & $\begin{array}{l}+21,5 \\
+20,0 \\
+27,5\end{array}$ & $\begin{array}{l}15,92 \\
16,16 \\
15,88 \\
14,98\end{array}$ & $\begin{array}{r}+1,5 \\
-0,2 \\
-5,9\end{array}$ & $\begin{array}{l}5,97 \\
6,12 \\
6,12 \\
5,72\end{array}$ & $\begin{array}{r} \\
+2,5 \\
+2,5 \\
+4,2 \\
\end{array}$ & $\begin{array}{l}50 \text { Sekunden pach Injektion Kräm- } \\
\text { pfe, Dauer } 30 \text { Sek. Shoek }(H)\end{array}$ \\
\hline $\begin{array}{l}6,12 \\
5,25 \\
5,79\end{array}$ & $\begin{array}{r}-14,2 \\
-5,4 \\
\end{array}$ & $\begin{array}{l}195 \\
251 \\
206\end{array}$ & $\begin{array}{r}+28,7 \\
+\quad 5,6\end{array}$ & $\begin{array}{l}14,52 \\
14,82 \\
13,92\end{array}$ & $\begin{array}{r} \\
+2,1 \\
-4,1 \\
\end{array}$ & $\begin{array}{l}5,58 \\
5,86 \\
5,64\end{array}$ & $\begin{array}{r}+5,0 \\
-0,7 \\
\end{array}$ & $\begin{array}{l}\text { 10 Sekanden nach Injektion Kräm- } \\
\text { pfe, Dauer } 10 \text { Sek. } 29 \text { Min. nach In- } \\
\text { jektion Herzstillstand. Shock (H) }\end{array}$ \\
\hline $\begin{array}{l}6,34 \\
5,86 \\
6,43\end{array}$ & $\begin{array}{r}-7,6 \\
+1,4\end{array}$ & $\begin{array}{l}184 \\
219 \\
266\end{array}$ & $\begin{array}{l}+19,0 \\
+44,6\end{array}$ & $\mid \begin{array}{l}15,26 \\
15,98 \\
15,36\end{array}$ & $\begin{array}{r}+4,7 \\
+0,6\end{array}$ & $\begin{array}{r}6,16 \\
5,90 \\
6,62\end{array}$ & $\begin{array}{r}-4,2 \\
+7,5 \\
\end{array}$ & $\begin{array}{l}\text { 30 Sekunden nach Injektion Kräm- } \\
\text { pfe, } 15 \text { Sek. dauert. } 22 \text { Min. nach In- } \\
\text { jektion Herzstillstand. Shock }(\#)\end{array}$ \\
\hline $\begin{array}{l}6,39 \\
6,13 \\
6,18 \\
6,24\end{array}$ & $\begin{array}{l}-4,1 \\
-3,3 \\
-2,3\end{array}$ & $\begin{array}{l}182 \\
200 \\
200 \\
220\end{array}$ & $\begin{array}{r}+9,9 \\
+9,9 \\
+20,9\end{array}$ & $\begin{array}{l}15,79 \\
16,20 \\
15,25 \\
15,12\end{array}$ & $\begin{array}{r}+2,6 \\
-3,4 \\
-4,2\end{array}$ & $\begin{array}{l}6,24 \\
6,27 \\
6,12 \\
6,02\end{array}$ & $\begin{array}{r}+0,5 \\
-1,9 \\
-3,5\end{array}$ & $\begin{array}{l}\text { 2 Min. nach Injektion besehleunigte } \\
\text { Atmung. Kein Krampf. Shock }(+)\end{array}$ \\
\hline
\end{tabular}

Serumeiweiss: DerSerumeiweissgehaltvorder Injektion war, wie oben bereits erwähnt, unter drei Blutarten im grossen ganzen im Lebervenenblut am geringsten gewesen. Unmittelbar nach dem Auftreten des Shocks nahm er im arteriellen Blut in sämtlichen Versuchen ab,im Pfortaderblut war das Eiweiss, ausgenommen den Versuch 1 mit Zunahme, in allen übrigen Versuchen vermindert, hingegen im Lebervenenblut wurde der Eiweissgehalt, abgesehen davon, dass er in Versuch 5 ziemlich deutlich abnahm, in Versuch 3 konstant blieb, in allen übrigen Versuchen vermehrt gefunden, und zwar war die Zunahme in Versuch 1 u. 4 grösser, in Versuch 2 u. 6 spärlich. Nach 15 Minuten war der Eiweissgehalt im arteriellen Blut in Versuch 4 u. 5 erhöht, in übrigen Versuchen noch weiter herabgesetzt. Im Pfortaderblut erfuhr der Eiweisspiegel in Versuch 3,4,5 und 6, der unmittelbar nach Shock- 
ausbruch abgesunken war, eine Erhöhung, die speziell in Versuch 3 u. 5 den Ursprungswert überschritt. Im Lebervenenblut erhöhte sich der Eiweissspiegel nur in Versuch 5, wo unmittelbar nach Shockausbruch ein Absinken aufgetreten war, schlug in allen übrigen Versuchen den Weg zur Herabsetzung ein, die jedoch gegenüber der Kontrolle geringergradig war. Nach 30 Minuten beherrschte im grossen und ganzen die Herebsetzung in drei Blutarten das Bild.

Aus dem oben Gesagten erhellt nämlich, dass unmittelbar nach Shockausbruch die Eiweisskonzentration des Lebervenenblutes zuerst ansteigt, nach 15 Minuten in den meisten Versuchen absinkt und dennoch in der Hälfte der Versuche den Ursprungswert übersteigt, und im Pfortaderblut unmittelbar nach Shockausbruch in der Mehrzahl der Fälle absinkt, nach 15 Minuten meistenfalls ansteigt, dass der Eiweissspiegel im arteriellen Blutmit dem Zeitablauf in den meisten Versuchen graduelle Herabsetzung erfährt, deren G Grad aber von demselben bei der Kontrolle nicht wesentlich abweicht.

\section{Besprechung.}

Über den Einfluss des Histamins auf den Blutzucker ist von jeher. viel diskutiert worden, allenfalls scheinen doch die Untersuchungen von vielen Autoren darin übereinzustimmen, dass beim Histamineinwirkung auf den Blutzucker je nach den Tierarten und jeweils angewandten Dosen und auch selbst bei Anwendung von weitgehend grösseren Dosen, durch welche der: Shock ausgelöst wird, je nach Graden des dadurch erzeugten Shocks der Blutzucker sich verschiedentlich verhält; immerhin dürfte soviel als sicher angesehen werden, dass bei Shock meist die Blutzuckervermehrung auftritt. Über den Mechanismus der hierbei auftretenden Veränderungen des Blutzuckers ist von verschiedenen Seiten vielfach umstritten, indem ein Teil der Autoren sie als zentral bedingt, ein ander Teil aber als peripher bedingt wissen will. Des weiteren lauten die Angaben über den Wirkungsmechanismus des Histamins recht divergierend, so nehmen eine Reihe Autoren eine lähmende Wirkung auf den Sympathicus, andere eine reizende Wirkung des Histamins auf den Parasympathicus an, während wieder andere Autoren behaupten, dass das Histamin direkt an glatter Muskulatur angreife.

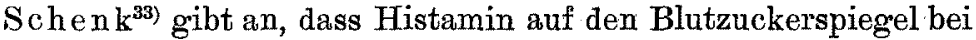
normalen Kaninchen keinen Einfluss ausübe, höchstwahrscheinlich auf den Sympathicus lähmend wirken dürfte. Katzenelbogen u. Ab- 
rams on ${ }^{55}$ äusserten sich, dass im mittelgradigen Shock die Hyperglykämie auftritt, während im hochgradigen Shock sie vermisst wird. Kellaway u. Cowell ${ }^{56}$ haben die Histaminhyperglykämie auf die Adrenalinausschüttung zurückgeführt. Chambers u. Thompson ${ }^{28)}$ haben das Verhalten des Glykogens und Blutzuckers im Histaminshock des Hundes studiert und gelangten zum Ergebnis, dass das Leberglykogen sowie das Herz- und Muskelglykogen auffallend abnehmen und der Blutzucker in erheblichem Masse zunimmt. Aber Kinoshita, ${ }^{30}$ ) welcher bei Kaninchen ebenfalls Hyperglykämie nachgewiesen hat, ist der Meinung, dass diese Hyperglykämie ihren Ursprung vom Leberglykogen nimmt und mit dem Muskelglykogen nichts zu tun hat. Der genannte Forscher gibt weiterhin der Ansicht Ausdruck, dass die Histaminhyperglykämie von der sympathischen bzw. Adrenalinhyperglykämie wesentlich abweicht und wohl durch die Erregung des Parasympathicus verursacht werden dürfte. A ok i ${ }^{34} \mathrm{u} . \mathrm{Ohm} \mathrm{i} \mathrm{i}^{35}$ sahen beim Kaninchen die Histaminhypoglykämie bestätigt; Maed ${ }^{32}{ }^{32}$ hat nach kleinen Mengen die Hypo-, nach grösseren Mengen Histamin die Hyperglylämie nachgewiesen; er meint, dass diese Hyperglykämie zentraler Natur sei, und der Impuls durch Vermittelung des Sympathicus zur Nebenniere fortgeleitet werde. $\mathrm{Zunz}$ u. La Barre, ${ }^{15}$ La Barr $e^{29}$ fanden bei Meerschweinchen, $\mathrm{O} \mathrm{kam}_{\star} \mathrm{oto}^{17}{ }^{17}$ bei Kaninchen die Hyperglykämie.

Bei der Durchsicht der literarischen Angaben über den Milchsäureumsatz im Histaminshock fällt auf, dass daraufhin gerichtete Untersuchungen nur von Chambers und Thompson ${ }^{28)}$ angestellt wurden, genannte Autoren sahen in den ersten $\frac{1}{2}$ Stunde den Milchsäuregehalt des Blutes um 130\% ansteigen.

Die in vorliegenden Versuchen festgestellte Tatsache, dass nicht allein im Lebervenen- sowie im Pfortaderblut, sondern auch im arteriellen Blut fast parallele Zuckervermehrung auftrat, legt den Gedanken nahe, dass das in Leber und Gedärmen gespeicherte Glykogen gespalten, mobilisiert wird und in den allgemeinen Kreislauf eindringt; immerhin dürfte doch die Mobilisation des Muskelglykogens nicht geleugnet werden.

Die intensivste Erhöhung des Milchsäurespiegels im Lebervenenblut dürfte wohl dahin erklärt werden, dass gleichzeitig mit gesteigerter Spaltung des Leberglykogens wegen der Funktionsstörung der Leber die Milchsäureresynthese sehr mangelhaft vor sich geht. Ein

55) Katzenelbogen u. Abramson, C. r. Soc. Biol. Paris, 1927, 97, 240.

56) Kellaw ag u. Cowe11, Journ. Physiol, 1922, 56, 20. 
Hinweis auf die Büngler ${ }^{57}$ Angabe, wonach bei Anwendung von grossen Mengen Hitamin der Stoffwechsel in der Leber in ähnlicher Weise, wie im anaphylaktischen Shock gehemmt werde, dürfte genügen, die Milchsäureanhäufung in der Leber zuzugeben.

Miyak $\mathrm{e}^{58}$ hat an Kaninchen die Untersuchung über die Histaminwirkung auf verschiedene Gefässe angestellt; diese Untersuchung: ergab, dass die Darmgefässe angeblich durch Histamin stark angegriffen wurden und an der Darmwand sich degenerative Veränderung durch die Stauung einstellte. Zu meinem Ergebnis übergehend, dürfte die Milchsäurezunahme im Pfortaderblut auf die durch die Durchblutungsstörung in der Leber bedingte sekundäre Zirkulationsstörung wie auch auf die primäre Zirkulationsstörung im Pfortadergebiet an sich bezogen werden.

Es wird nun die Frage aufgeworfen: Woher kommt es denn, dass unmittelbar nach dem Auftreten des Shocks die Milchsäure im arteriellen Blut vorübergehend abnimmt? Es haben Dale u. Làidlaw, ${ }^{59}$, Cloetta u. Anderes $\mathrm{s}^{60}$ und $\mathrm{Ab} \mathrm{e}^{61)}$ an Katzen und Kaninchen, Baehr u. Pick ${ }^{62}$ an Meerschweinchen, McDowall, ${ }^{63)} \mathrm{Osawa} a^{64 j}$ an Katze, Fühner u. Starling ${ }^{65}$ und Manwaring, Monaco u. Marino $0^{66)}$ am Hund, Miyake $e^{58}$ und Dixon u. Hoyle $e^{67}$ an Kaninchen im Durchströmungsversuch an überlebender Lunge den Nachweis erbracht, dass das Histamin die Lungengefässe zu kontrahieren vermag. Sa to ${ }^{68}$ hat röntgenologisch im Histaminshock des Kaninchens in eben ähnlicher Weise wie im anaphylaktischen Shock recht beträchtliche Veränderungen der Lungenarterien nachgewiesen.

Dass das Histamin auch durch seine periphere Wirkung die Bronchialmuskulatur zur Kontraktion bringt, ist durch Untersuchungen von Dale u. Laidlaw, ${ }^{59}$ Baehru. Pick, ${ }^{62}$ Titone, ${ }^{69}$ Müller, Salomon u. Zuelzer ${ }^{70)}$ u. a. erwiesen worden. Im Histaminshock stellt sich also

57) B üngle r, Frankfurt. Ztschr. Pathol., 1932, 44, 1.

58) Miyake, Nippon Naibumpigakkai Zasshi, 1930, 6, 66.

59) Da le u. L aidla w, Journ. Physiol., 1910, 41, 318.

60) Cloetta u. Anderes, Arch. f. exp. Path. u. Pharm., 1914, 76, 125.

61) A be, Tohoku Igaku Zasshi, 1919, 4, 19.

62) Ba ehr u. Pick, Arch. f. exp. Path. u. Pharm., 1913, 74, 65.

63) Me Dow a 11, Journ. Physiol., 1923, 57, 146.

64) Os aw a, Arch. f. exp. Path. u. Pharm., 1930, 156, 309.

65) Fühneru. Starling, Journ. Physiol., 1913, 47, 286.

66) Manwaring, Mona co u. Marino, Journ. Immunol., 1923, 8, 217.

67) Dix on u. Hoyle, Journ. Physiol., 1930, 70, 1.

68) Sa to, Nippon Naikagakkai Zasshi, 1937, 25, 409.

69) Tito ne, Arch. f. ges. Physiol., 1914, 155. 77.

70) Mülle r, Sa lo mo n u. Zuelzer, Arch. P. exp. Path. u. Pharm., 1932, 164, 441. 
neben der Verengerung der Lungengefässe das Zusammenziehen der Brochialmuskulatur ein, welches seinerseits das Kaliber der Gefässe einengt und somit auch die Störung des Lungenkreislaufes mit sich bringt.

Dass die Blutmilchsäure physiologischerweise im Lungenkreislauf teilweise beseitigt wird, ist schon die erwiesene Tatsache, worauf ich bereits in der I. Mitteilung ${ }^{1)}$ hingewiesen habe. Nach Knippin . $^{\text {11) }}$ und Alpern, Simonson, Sirkina u. Tutkiewits ch $^{72}$ soll sich beim Zustandekommen der Kreislaufstörung bzw. Anoxämie in der Lunge die oxydative Milchsäurebeseitigung ausserordentlich vermehren. Darauf beruhen muss aller Wahrscheinlichkeit nach die Milchsäureabnahme im arteriellen Blut im Histaminshock.

Es gibt eine recht grosse Anzahl von experimentellen Beobachtungen, die sich mit dem Wasseraustausch im Histaminshock befassen.

So fallen z.B. in der Literatur folgende Beschreibungen anf: Dale u. Laid$1 \mathrm{a} \mathrm{w}^{4}$ haben darauf aufmerksam gemacht, dass im Histaminshock der Katze eine plötzliche Oligämie auftritt, die durch Verlangsamung der Blutströmung in den kleinsten peripheren Gefässen einerseits und durch den Austritt des Plasmas aus der Blutbahn anderseits bedingt sei, und diese beiden Momente führten den Blutdrucksturz und die Eindickung des Gesamtblutes herbei. In jüngster Zeit haben Eppingeru. Leuchtenberge $\mathrm{r}^{25}$ an Hunden die Beobachtung gemacht, dass durch Histamininjektion der Hämoglobingehalt sich erhöht, der Eiweissgehalt aber fast unverändert bleibt; genannte Autoren sind der Meinung, dass dieser Befund wahrscheinlich darauf beruhen dürtte, dass infolge zugleich erfolgender beträ,chtlicher Abnahme der zirkulierenden Blutmenge das Blutplasma die Blutgefässe verlässt; somit stimmen sie den Ansichten von letztgenannten Autoren bei. Ku shin sk $\mathrm{y}^{73)}$ hat hervorgehoben, dass im protrahierten Histaminshock beim Hund das Serumeiweiss keine nennenswerte Veränderung erfährt. De Ton $\mathrm{i}^{\text {74) }}$ fand beim Hund im Histaminshock, dass der refraktometrische Eiweisswert in der Hälfte ansteigt, in der anderen Hälfte der Fälle absinkt, D ér e r u. S teffa $\mathrm{n} u \mathrm{t}_{\mathrm{i}}^{\mathrm{h} 1}$ berichteten, dass bei Histaminkatzen das Gesamtblut eingedickt ist, die Serumeiweisskonzentration dessen ungeachtet abnimmt. Puddu u. Vit to ri $0^{52}$ konnten zeigen, dass beim Hund der Hämoglobingehalt erhöht ist, der Eiweisswert unverändert bleibt, wobei die Kapillarwand für alle Eiweisskörper, besonders aber für Albumine durchlässig ist.

Des weiteren hat Dal $\mathrm{e}^{\mathbf{8} \text { ? }}$ bei Katze, Ma ed a ${ }^{501}$ bei Meerschweinchen einen Hämoglobinanstieg konstatiert. Underhill u. Roth ${ }^{75}$ äusserten sich, dass beim

71) Knipping, Klin. Wsehr., 1934, 1449.

72) Alpern, Simonson, Sirkina u. Tutkiewitsch, Arch. f. ges. Physiol., $1935,235,554$.

73) Kus hinsky, Ztschr. exp. Med., 1928, 64, 563.

74) De Toni, Boll. Soc. ital. Biol. sper., 1928, 3, 87.

75) Underhill u. Roth, Jonrn. Biol. Chem., 1922, 54, 607. 
Kaninchen die Blutkonzentration anscheinend kaum beeinflusst wird. Sato ${ }^{49}$ gibt an, dass zwar der Grad der Bluteindickung nicht so erheblich ist, die nachweisbare Erhöhung derselben aber 5-15 Minuten nach Histamininjektion stets unfehlbar eintritt. A d le rsberg $\mathrm{u} . \mathrm{Pa} \mathrm{l}^{53)}$ haben im Histaminshock des Hundes mit der Methode der Quaddelbildung eine Störung des Wasseraustausches nachgewiesen und diese anf die Schädigung der Leberfunktion zurückgeführt. Ma nwaring, Monaco u. Marino $0^{76}$ haben den Beweis geführt, dass der Haupteffekt des Histamins sich in plötzlicher Permeabilitätssteigerung der Kapillaren äussert, wobei Gewebe ödematös getränlkt sind.

In bezug auf das Verhalten der Hämoglobin- und Eiweissveränderungen im Histaminshock finden die Ansichten der meisten Autoren, wie oben geschildert, im groben im Hämoglobinanstieg und in der Stetigkeit des Eiweissgehaltes Übereinstimmung, obzwar zugregeben werden muss, dass je nach den Tierarten Abweichungen resultieren können.

In vorliegendem Versuch wurde festgestellt, dass im arteriellen Blut die Erhöhung des Hämoglobingehaltes auftrat, wohingegen die Eiweisskonzentration nicht anstieg, sondern fast gleiche Verhältnisse wie im Kontrollversuch aufwies. Wenn man deshalb nur allein das Verhalten des arteriellen Blutes in Erwägung zöge, so könnte man wohl annehmen, dass im Histaminshock, wie Dale u. L aidlaw sowie Eppinger u. Leuchtenberger hervorheben, das Plasma die Blutgefässe verlässt und in die Gewebe übertritt, indessen dürften die Verhältnisse im Pfortadergebiet, vor allem aber in der Leber keineswegs so einfach hingenommen werden. Dass unmittelbar nach Shockausbruch die Hämoglobin- und Eiweisskonzentration im Lebervenenblut gegenuiber den Werten in zwei in die Leber hineinziehenden Blutarten erhöhte Werte aufwiesen, und dass, was besonders interessant erscheint, der Eiweissgehalt des Lebervenenblutes, der anfänglich den niedrigsten Wert dargeboten hatte, nachher denselben in zwei letzt genannten Blutarten uberschritt und so die Oberhand gewann, wird höchstwahrscheinlich darauf bezogen werden, dass die Leber dem Blute aufs intensivste Wasser entzog und ödematös anschwoll; keinesfalls aber dürfte es sich hjerbei meines Erachtens um denWasserverlust allein handeln. Denn im Hinblick auf den Umstand, dass ein Vergleich von prozentischer Zu- oder Abnahme des Eiweiss- nnd Hämoglobingehaltes im Lebervenenblut die gerade zu Hälfte miteinander abwechselnde Überlegenheit und Minderwertigkeit der beiden Grössen ergibt, kann mit Sicherheit angenommen werdén, dass hierbei die bald eiweiss325.

76) Manwaring, M o naco u. Marino, Arch. f. exp. Path. u. Pharm., 1931, 161, 
ärmere, bald eiweissreichere Flüssigkeit in das Lebergewebe eintritt, während aber anderseits mitunter die Eiweissmolekïle in die Blutgefässe mobilisiert werden. Das sind nun freilich Dinge, die sicherlich nicht ohne weiteres erledigt werden können.

Betreffend die sich hier abspielenden Veränderungen dürfte die Auffassung gerechtfertigt sein, dass das Histamin, wie Eppinger hervorhebt, die Schädigung der Kapillarendothelien bewirkt und somit deren Permeabilitätssteigerung herbeiführt, und dass es auch andererseits meines Erachtens die Schädigung der Leberparenchymzellen hervorruft, wodurch die Eiweisskonstitution derselben Veränderungen erfährt, dass möglicherweise auch das Depoteiweiss zur Mobilisation veranlasst wird.

Im Ablauf von 15 Minuten nach Histamininjektion wurde die Bluteindickung noch beibehalten, nach 30 Minuten aber war das Hämoglobin auf den beinahe gleichen Wert wie der im Kontrollversuch herabgesetzt; dieser Befund steht im guten Einklang mit dem Bericht von Sato, ${ }^{49)}$ wonach die Bluteindickung nur kurze Zeit nach dem Auftreten des Shocks andauert. Die unmittelbar nach Shockausbruch eingetretene Bluteindickung des Pfortaderblutes dürfte, wie Manwaring und seine Mitarbeiter angeben, durch ödematöse Anschwellung der Gedärme und Wasserentzug verursacht sein; doch ist es bemerkenswert, dass die Eiweisskonzentration unmittelbar nach shockausbruch herabgesetzt und erst nach 15 Minuten erhöht war.

Venn man nun oben erwähnte Vèränderungen von Milchsäure, Zucker, Hämoglobin und Eiweiss mit entsprechenden Veränderungen im anaphylaktischen Shock vergleicht, so ergibt sich folgendes: Betreffs der Veränderuing der Milchsäure besteht kein nennenswerter Unterschied zwischen den beiden Shockformen. Während der Zuckerspiegel im anaphylaktischen Shock im Lebervenenblut deutliche Erhöhung aufweist, im arteriellen und Pfortaderblut hingegen fast konstant bleibt und in manchen Versuchen ausnahmsweise transitorisch herabgesetzt ist, erhöht sich der Zuckerspiegel im Histaminshock in drei Blutarten parallel.

In bezug auf die Hämoglobinveränderung lässt sich kein grosser Unterschied zwischen den beiden Shockarten erkennen. In Serumeiweissveränderung tritt die Differenz zwischen den beiden Shockarten ganz auffallend zutage. Während im anaphylaktischen Shock nämlich das Serumeiweiss in drei Blutarten, insbesondere im Lebervenenblut deutlich zunimmt, und zudem die prozentische Zunahme grösser ist und länger andauert, nimmt das Serumeiweiss im Histaminshock hin- 
gegen nur im Lebervenenblut zu, und diese prozentische Zunahme ist geringer und von äusserst kurzer Dauer, ja tritt in etlichen Versuchen sogar eine erhebliche Abnahme auf ; im arteriellen und Pfortaderblut können Eiweisswerte im ganzen genommen, als beinahe konstant angesehen werden.

Wie aus dem oben Gesagten zur Gentige hervorgeht, tritt eine markante Differenz zwischen den beiden Shockarten insofern, als es den Blutzucker- und Eiweissumsatz betrifft, in Erscheinung, besonders hinsichtlich des letzteren ist die Reagierbarkeit der Leber im anaphylaktischen Shock höhergradig.

Dass die Sensibilisierung des Organismus mit physikochemischer Veränderung der Zellstruktur verbunden ist, ist von Doer ${ }^{77}$ und Tonietti ${ }^{78)}$ u. a. erwiesen worden. Wid a ${ }^{79)}$ hat den anaphylaktischen Shock als eine kolloidale Gleichgewichtsstörung aufgefasst, Abderhalden u. Wertheimer ${ }^{80)}$ sowie Berger ${ }^{81}$ haben nach Erst- und Reinjektion mit Hilfe der Refraktometrie die Hyperproteinoplasie konstatiert und die Verschiebung des betreffenden Brechnungswertes auf physikochemische Änderungen der gelösten Stoffe, insonderheit der Eiweisskörper zurückgeführt. Nach Berger soll die Hyperproteinämie beim anaphylaktischen Shock mit grosser Wahrscheinlichkeit auf echter Vermehrung des Bluteiweisses durch gesteigerte Abgabe von Zellproteine im Blut beruhen, keineswegs durch eine Bluteindickung oder durch verminderten Eiweissverbrauch verursacht werden.

Nach alledem ist es leicht verständlich, dass im anaphylaktischen Shock, wo die Gewebszellen tiefgreifende physikochemische Veränderungen aufweisen, die Leber, das Zentralorgan für den Gesamtstoffwechsel aufs intensivste in Mitleidenschaft gezogen werden muss.

Hashim oto u. $\mathrm{Pick}^{82)}$ haben den Nachweis erbracht, dass durch einmalige parenterale Applikation von äusserst geringer Menge körperfremden Eiweisses eine gewaltige Organproteolyse stattfindet, und dass diese Veränderung in der Leber besonders ausgesprochen zum Vorschein kommt, und zwar derart, 1/5-1/4 von Lebereiweiss in die Spaltprodukte umgewandelt sind. Fenyvessy u. Freund, ${ }^{\left.8_{3}\right)}$ Bieling, Gottsehalk u. Jsaac, ${ }^{84}$ Fischler ${ }^{85}$ haben aus im anaphy-

77) Doerr, Erg. Hyg., 1922, 5, 71.

78) Tonietti, Ztschr. exp. Med., 1925, 45, 1.

79) Widal, Presse méd., 1920, I, 181.

80) Abderhalden u. Wertheimer, Arch. f. ges. Physiol., 1922, 197, 85.

81) Berger, Ztschr. exp. Med., 1922, 28, 1.

82) Hashimoto u. Piek, Areh. f. exp. Path. u. Pharm., 1914, 76, 89.

83) Fenyvessy u. Freund, Biochem. Ztschr., 1919, 96, 223.

94) Bieling, Gottschalk u. J s a c, Klin. Wschr., 1922, 1560.

85) Fis chl er, Physiologie u. Pathologie der Leber, 2. Aufl., Berl. 1925. 
lalstischen Shock auftretender Zunahme des Rest-N in der Leber die Veränderung des intrahepatischen Eiweissumsatzes erkannt, auch $\mathrm{Sanada} \mathrm{a}^{867}$ hat am Lebergewebe erhebliche Zunahme des Gesamt- $\mathrm{N}$ und Rest- $\mathrm{N}$ nachgewiesen. Manwaring ${ }^{2}$ fand beim anaphylaktischen Shock beträchtliche Zunahme des Nettgewichtes der Leber, Ma utner u. Pi $\mathrm{ck}^{87)}$ konstatierten die Leberstauung durch Verschluss der V. hepatica. Martin u. Croizat, $\left.{ }^{88}\right)$ Hajós u. Németh ${ }^{893}$ sahen starke degenerative Veränderung der Leberzellen, W e i ${ }^{26)} \mathrm{u}, \mathrm{B}$ ü $\mathrm{ugl} \mathrm{er}^{\mathrm{s0}}$ fanden hochgradige Hyperämie der Leber; V elard $i^{9 l)}$ Konnte zeigen, dass die Leber im anaphylaktisehen Shock Farbstoffe stark aufnimmt.

Aus obigen Ergebnissen geht klar hervor, dass die Leber im anaphylaktischen Shock markante Veränderungen erleidet.

Dass auch beim Histaminshock die Leber beeinträchtigt wird, ist durch Untersuchungen von Mautner u. Pick, ${ }^{7}$ Eppinger u. Leuchtenberger, ${ }^{25)}$ die alle das Auftreten starker Hyperämie der Leber nachweisen konnten, klargestellt worden. Indes haben Manwaring, Chilcote, Clark u. Mona $\mathrm{co}^{10}$ die Beobachtung gemacht, dass beim Hund mit ausgeschalteter Leber nicht der anaphylaktische und Peptonshock, wohl aber der wenn auch nicht typische schwere Histaminshock auftritt; auf Grund dieser Beobachtung sprechen genannte Autoren die Meinung dahin aus, dass im Histaminshock der Leber jene dominierende Stellung; wie sie im anaphylaktischen Shock beobachtet wird, nicht zukommt.

Nach obigen Angaben sowiemeinen Versuchsergebnissen ist wohl anzunehmen, dass im anaphylaktischen Shock die Gewebszellen, im besonderen Zellen der Leber und des Splanchnikusgebietes tiefgreifende anhaltende physikochemische Veränderungen erleidet, wodurch die Eiweisskörper in kolossalen Mengen ins Blut übertreten. Im Histaminshock hingegen dürfte die Schädigung der Leberzellen relativ geringfügig sein. In vorliegenden Versuchen nahm der Eiweissgehalt im Lebervenenblut in geringem Masse zu; es fand zwar zuweilen die Mobilisation der Eiweissstoffe statt, die aber überaus geringgradig und temporärer Natur war.

Im übrigen ist über die Differenz in den im anaphylaktischen, Histamin- sowie Peptonshock auftretenden Veränderungen des kolloidosmotischen Drucks des Bluteiweisses in drei, in der Leber zirkulie-

86) Sanad a, Tohoku.Journ. Exp. Med., 1936, 29, 212.

87) Ma utner u. Pick, Münch. med. Wschr., 1915, 1141.

88) Martin u. Croizat, C. r. Soc. Biol. Paris, 1927, 97, 95.

89) Hajós u. Németh, Ztschr. exp. Med., 1925, 45, 513.

90) B üngle r, Ibid., 1931, 75, 223.

91) Ve1ardi, Rif. med., 1923, 39, 1161; rel. in Kongzbl. f. inn. Med., 1924, 34, 137. 
renden Blutarten in gemeinschaftlicher Arbeit von mir und $S$ a n a d $a^{\text {92) }}$ wie auch in meiner Mitteilung ${ }^{93}$ andernorts berichtet worden.

\section{Peptonshock.}

Es wurde Pepton (Teruuchi) im Mengenverhältnis von 20\% in physiologischer Kochsalzlösung aufgelöst und nach Sterilisierung in Ampulle aufbewahrt; zwecks Auslösung des Peptonshocks wurden davon 1,5 ccm pro kg Körpergewicht dem Versuchstier injiziert. Bei Anwendung von gleichen Dosen traten dadurch hervorgerufene Shocksymptome, wie auch viele Autoren angeben, je nach den Individuen

\section{Tabelle 3 .}

Veränderungen des Blutmilchsäurespiegels in der Leber beim Peptonshock.

\begin{tabular}{|c|c|c|c|c|c|c|c|c|c|}
\hline \multirow{2}{*}{ 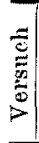 } & \multirow{2}{*}{$\begin{array}{c}\text { Geschlecht } \\
\text { u. } \\
\text { Körperge- } \\
\text { wicht } \\
\text { (kg.) }\end{array}$} & \multirow{2}{*}{$\begin{array}{c}\text { Zeit der } \\
\text { Blutent- } \\
\text { nahme } \\
\text { (Min.) }\end{array}$} & \multicolumn{2}{|c|}{ A. carotis } & \multicolumn{2}{|c|}{ V. portae } & \multicolumn{2}{|c|}{ V. hepatica } & \multirow{2}{*}{ Bemerkungen } \\
\hline & & & $\underset{\mathrm{dl}}{\mathrm{mg} /}$ & $\underset{\%}{\text { Diff. in }}$ & $\underset{\mathrm{di}}{\mathrm{mg} /}$ & $\begin{array}{c}\text { Diff. in } \\
\%\end{array}$ & $\underset{\mathrm{dl}}{\mathrm{mg} /}$ & $\int_{\%}^{\text {Diff }}$ & \\
\hline 1 & $\stackrel{\hat{0}}{1,80}$ & $\begin{array}{l}\text { Vor d. Inj. } \\
\text { Nach } 5 \text { Min. } \\
\quad, \quad 15,\end{array}$ & $\begin{array}{l}36,00 \\
36,64 \\
50,14\end{array}$ & $\begin{array}{r}+1,8 \\
+39,3\end{array}$ & $\begin{array}{l}32,79 \\
40,50 \\
59,14\end{array}$ & $\begin{array}{l}+23,5 \\
+80,3\end{array}$ & $\begin{array}{l}27,64 \\
40,14 \\
60,43\end{array}$ & $\begin{array}{r}+45,2 \\
+118,6\end{array}$ & $\begin{array}{l}1 \text { Min. nach Injektion } \\
\text { Krämpie, Dauer } 15 \\
\text { Sekunden. } 28 \frac{1}{4} \text { Min. } \\
\text { Herzstillstand. Shook } \\
\text { (H) }\end{array}$ \\
\hline 2 & $\begin{array}{c}\hat{\sigma} \\
1,92\end{array}$ & $\begin{array}{l}\text { Vor d. Inj. } \\
\text { Nach } 5 \text { Min. } \\
\begin{aligned}, \quad 15, \\
, \quad 30 "\end{aligned}\end{array}$ & $\begin{array}{l}24,43 \\
24,43 \\
25,07 \\
31,50\end{array}$ & $\begin{array}{l} \pm 0 \\
+\mathbf{2}, 6 \\
+28,9\end{array}$ & $\begin{array}{l}23,14 \\
29,57 \\
23,14 \\
32,14\end{array}$ & $\begin{array}{l}+27,8 \\
\pm 0 \\
+38,9\end{array}$ & $\begin{array}{l}21,21 \\
30,86 \\
30,86 \\
40,50\end{array}$ & $\begin{array}{r}+ \\
+\quad 45,5 \\
+\quad 45,5 \\
+\quad 90,9\end{array}$ & $\begin{array}{l}3 \text { Min: nach Injektion } \\
\text { leichtgradiger Krampl. } \\
\text { Shook }(+)\end{array}$ \\
\hline 3 & $\stackrel{\text { 今 }}{2,05}$ & $\begin{array}{l}\text { Vor d. Inj. } \\
\text { Nach } 3 \text { Min. } \\
\quad, 15 \text {, }\end{array}$ & $\begin{array}{l}38,57 \\
38,57 \\
49,50\end{array}$ & $\begin{array}{l} \pm 0 \\
+28,3\end{array}$ & $\mid \begin{array}{l}35,36 \\
51,43 \\
52,07\end{array}$ & $\begin{array}{r}+45,4 \\
+47,2\end{array}$ & $\begin{array}{l}34,71 \\
41,14 \\
52,71\end{array}$ & $\begin{array}{r} \\
+\quad 18,5 \\
+\quad 51,8\end{array}$ & $\begin{array}{l}1_{3}^{2} \text { Min. nach Injektion } \\
\text { Krämpfe, Dazer } 10 \\
\text { Sekunden. } 27 \text { Min. nach } \\
\text { Injektion Herzstillstand. } \\
\text { Shock }(+\#)\end{array}$ \\
\hline 4 & $\begin{array}{c}\hat{\delta} \\
1,85\end{array}$ & $\begin{array}{l}\text { Vor a. Inj. } \\
\text { Nach ô Min. } \\
\Rightarrow \quad \mathbf{1 5}, \\
\Rightarrow \quad 30 "\end{array}$ & $\begin{array}{l}27,00 \\
23,14 \\
36,00 \\
32,79\end{array}$ & $\begin{array}{r}-14,3 \\
+33,3 \\
+21,4\end{array}$ & $\begin{array}{l}25,71 \\
30,86 \\
37,29 \\
26,39\end{array}$ & $\begin{array}{r}+20,0 \\
+45,0 \\
+\quad 2,6\end{array}$ & $\begin{array}{l}18,64 \\
21,85 \\
23,14 \\
30,21\end{array}$ & $\begin{array}{l}+17,2 \\
+\quad 24,1 \\
+\quad 62,1\end{array}$ & $\begin{array}{l}\text { Nur beschlennigte At- } \\
\text { mung nach Injektion. } \\
\text { Shock }( \pm)\end{array}$ \\
\hline 5 & 2 우 & $\begin{array}{l}\text { Vor d. Inj. } \\
\text { Nach } 5 \text { Min. } \\
\Rightarrow \quad 15 \quad " \\
" \quad 30 "\end{array}$ & $\begin{array}{l}37,29 \\
38,57 \\
45,64 \\
59,14\end{array}$ & $\begin{array}{r} \\
+3,4 \\
+22,4 \\
+58,6\end{array}$ & $\begin{array}{l}34,07 \\
40,50 \\
46,29 \\
66,85\end{array}$ & $\begin{array}{l}+19,9 \\
+35,9 \\
+96,2\end{array}$ & $\begin{array}{l}29,57 \\
36,64 \\
42,43 \\
67,49\end{array}$ & $\begin{array}{r}+23,9 \\
+\quad 43,5 \\
+128,2\end{array}$ & $\begin{array}{l}\text { 2 Min. nach Injektion } \\
\text { Krämpfe, Dauer } 12 \\
\text { Sekunden. Shock }(+)\end{array}$ \\
\hline 6 & $\begin{array}{c}\delta \\
1,88\end{array}$ & 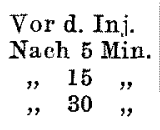 & $\begin{array}{l}28,29 \\
28,93 \\
33,43 \\
45,64\end{array}$ & $\begin{array}{l}+2,3 \\
+18,2 \\
+61,3\end{array}$ & $\begin{array}{l}24,43 \\
31,50 \\
31,50 \\
47,57\end{array}$ & $\begin{array}{r}+28,9 \\
+28,9 \\
+94,7\end{array}$ & $\left|\begin{array}{l}23,79 \\
23,14 \\
31,50 \\
50,1 \hat{4}\end{array}\right|$ & $\begin{array}{r}2,6 \\
+\quad 32,4 \\
+110,7\end{array}$ & $\begin{array}{l}\text { Unruhig, besehleunigte } \\
\text { Atmung. Kein Krampf. } \\
\text { Shock }(+)\end{array}$ \\
\hline
\end{tabular}

92) Tsuge u. Sanada, Tohoku Journ. Exp. Med., 1988, 32, 587.

93) Tsuge, Wird baldigst in dieser Joumal publiziert. 
ausserordentlich verschieden stark zutage. Im grossen und ganzen jedoch traten 10 Sek.-2 Min. nach der Injektion Krämpfe auf, dabei angetroffene Zustände wichen nicht so sehr von denselben im anaphylaktischen und Histaminshock ab.

Milchsäure: An 6 Kaninchen gewonnene Versuchsergebnisse sind in Tab. 3 wiederg'egeben.

Unmittelbar nach Shockausbruch wurde der Milchsäuregehalt im arteriellen Blut, ausgenommen den Versuch 4 mit deutlicher Abnahme, entweder unverändert oder ein wenig vermehrt gefunden. Im Pfortader- und Lebervenenblut zeigte die Milchsäure, mit Ausnahme des Versuchs 6 , wo eine Abnahme im Lebervenenblut erfolgte, in allen übrigen Versuchen erhebliche Zunahme; die prozentische Zunahme war im Lebervenenblut grösser. Nach 15 Minuten trat erhebliche Erliöhung im arteriellen Blut auf, nur in Versuch $2 \mathrm{kam}$ der Milchsäurespiegel im Pfortaderblut, der anfänglich gesteigert war, zum Anfangswert zurück, in übrigen Versuchen wurden in drei Blutarten weitere Erhöhungen angetroffen, und zwar besonders auffallend im Lebervenenblut. Nach 30 Minuten wurde, abgesehen von Versuch 4, wo eine Herabsetzung: im arteriellen und Pfortaderblut auftrat, deutlicher Anstieg: angetroffen; im Lebervenenblut ging: der Milchsäurespiegel weiter fortgesetzt in die Höhe.

In direktem Anschluss an den Shockausbruch blieb der Milchsäuregehalt nämlich im arteriellen Blut fast unverändert, so dass er gegenüber dem Kontrollwert fast keinen Unterschied aufwies, im Pfortader- und Lebervenenblut setzte deutliche Erhöhung ein; mit dem Zeitverlauf aber stellten sich weitere Erhöhungen in drei Blutarten ein, bis der Milchsäurespiegel des Lebervenenblutes mit dem Zeitablauf immer höher stieg und schliesslich denselben des Pfortaderblutes übertraf (Fig. 3). Es war dies ein Beweis für die Milch-

Fig. 3. Veränderungen des Milchsäuregehaltes des arterillen, des Pfortader- und des Lebervenenblutes beim Peptonshock (Versuch 1).

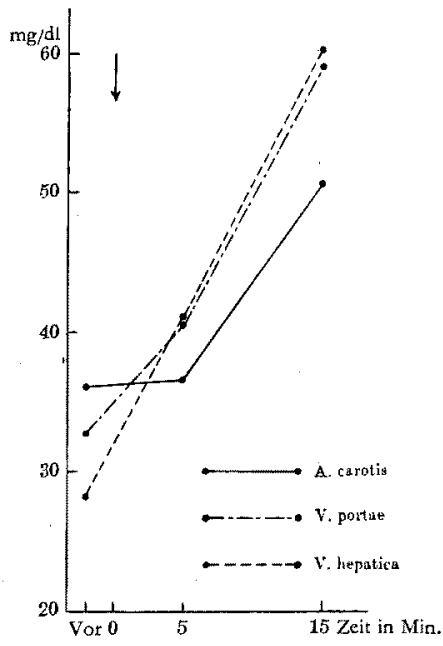
säureanhäufung in der Leber. 
Zucker: An 6 Kaninchen erhaltene Ergebnisse von Zucker-, Hämoglobin- und Eiweissbestimmungen sind in Tab. 4 zusammengestellt.

Unmittelbar nach Shockausbruch erfuhr der Zuckerspiegel in drei Blutarten gemeinsame Erhöhung, die in Versuch 2, 3 u. 4 besonders ausgesprochen war. Und diese Erhöhungen hielten fast gleichen Schritt in drei Blutarten, abgesehen davon, dass die Erhöhung im arteriellen Blut in Versuch 4 und im Pfortaderblut in Versuch 5 u. 6 deutlicher erfolgte. Nach 15 Minuten wurde weitere Erhöhung nur in Versuch 6 ermittelt, in übrigen Versuchen fand sich der Auf- und Abstieg in geringem Grad in drei Blutarten. Der Zuckergehalt des Lebervenenblutes zeigte stets den höchsten Wert. Nach 30 Minuten sank der Zuckerspiegel nur in Versuch 1 in drei Blutarte unnter den Ausgangs-

Tabelle

Veränderungen des Blutzucker-, Hämoglobin- u.

\begin{tabular}{|c|c|c|c|c|c|c|c|c|c|c|c|c|}
\hline \multirow{3}{*}{$\begin{array}{l}\text { Ver- } \\
\text { such }\end{array}$} & \multirow{3}{*}{$\begin{array}{c}\text { Gesch- } \\
\text { lecht } \\
\text { u. } \\
\text { Körpex- } \\
\text { gewicht } \\
\text { (kg.) }\end{array}$} & \multirow{3}{*}{$\begin{array}{c}\text { Zeit der } \\
\text { Blutent- } \\
\text { nahme } \\
\text { (Min.) }\end{array}$} & \multicolumn{6}{|c|}{ A. carotis } & \multicolumn{4}{|c|}{ V. portae } \\
\hline & & & \multicolumn{2}{|c|}{ Zucker } & \multicolumn{2}{|c|}{$\mathrm{Hb}$} & \multicolumn{2}{|c|}{$\begin{array}{c}\text { Serumei- } \\
\text { weiss }\end{array}$} & \multicolumn{2}{|c|}{ Zucker } & \multicolumn{2}{|c|}{$\mathrm{Hb}$} \\
\hline & & & $\underset{\mathrm{dl}}{\mathrm{mg} /}$ & $\begin{array}{l}\text { Diff. } \\
\text { in } \%\end{array}$ & $g / \mathrm{dl}$ & $\begin{array}{l}\text { Diff. } \\
\text { in } \%\end{array}$ & $\%$ & $\begin{array}{l}\text { Diff. } \\
\text { in } \%\end{array}$ & $\underset{\mathrm{dl}}{\mathrm{mg} /}$ & $\begin{array}{l}\text { Diff. } \\
\text { in } \%\end{array}$ & g/dl & $\begin{array}{l}\text { Diff. } \\
\text { in } \% 6\end{array}$ \\
\hline 1 & $\begin{array}{c}\hat{\delta} \\
2,11\end{array}$ & $\begin{array}{c}\text { Vor d. Inj. } \\
\text { Nach } 5 \text { Min. } \\
\Rightarrow 15 \quad " \\
" 30 "\end{array}$ & $\begin{array}{l}206 \\
222 \\
221 \\
176\end{array}$ & $\begin{array}{r}+7,8 \\
+7,3 \\
-14,6\end{array}$ & $\begin{array}{l}14,67 \\
14,67 \\
14,70 \\
14,52\end{array}$ & $\begin{array}{l} \pm 0 \\
+0,2 \\
-1,0\end{array}$ & $\begin{array}{l}\mathbf{5}, 90 \\
5,88 \\
5,72 \\
5,90\end{array}$ & $\begin{array}{l}-0,3 \\
-3,0 \\
\pm 0\end{array}$ & $\begin{array}{l}197 \\
215 \\
215 \\
134\end{array}$ & $\begin{array}{r}+9,1 \\
+9,1 \\
-32,0\end{array}$ & $\begin{array}{l}14,52 \\
14,52 \\
14,49 \\
14,39\end{array}$ & $\begin{array}{l} \pm 0 \\
\pm 0,2 \\
-0,9\end{array}$ \\
\hline 2 & $\begin{array}{c}\hat{o} \\
1,86\end{array}$ & $\begin{array}{c}\text { Vor d. Inj. } \\
\text { Nach } 5 \text { Min. } \\
\Rightarrow 15 \quad " \\
" 30 ",\end{array}$ & $\begin{array}{l}175 \\
217 \\
232 \\
217\end{array}$ & $\begin{array}{l}+24,0 \\
+32,6 \\
+24,0\end{array}$ & $\begin{array}{l}14,96 \\
14,80 \\
14,24 \\
13,64\end{array}$ & $\left|\begin{array}{r} \\
-1,1 \\
-4,8 \\
-8,8\end{array}\right|$ & $\begin{array}{l}6,32 \\
5,90 \\
5,68 \\
5,90\end{array}$ & $\begin{array}{r}-6,6 \\
-10,1 \\
-6,6\end{array}$ & $\begin{array}{l}173 \\
210 \\
229 \\
188\end{array}$ & $\begin{array}{r}+21,4 \\
+28,3 \\
+8,7\end{array}$ & $\left|\begin{array}{l}14,80 \\
14,80 \\
14,30 \\
13,94\end{array}\right|$ & $\begin{array}{l} \pm 0 \\
-\mathbf{3}, 4 \\
-\tilde{\mathbf{5}}, 8\end{array}$ \\
\hline 3 & $\begin{array}{c}\hat{\delta} \\
1,70\end{array}$ & $\begin{array}{c}\text { Vor d. Inj. } \\
\text { Nach } 5 \text { Min. } \\
\Rightarrow \text { I5 " } \\
" 30 "\end{array}$ & $\begin{array}{l}216 \\
268 \\
259 \\
266\end{array}$ & $\begin{array}{l}+24,1 \\
+19,9 \\
+23,1\end{array}$ & $\begin{array}{l}16,48 \\
16,48 \\
16,24 \\
16,21\end{array}$ & $\left|\begin{array}{l} \pm 0 \\
-1,4 \\
-1,6\end{array}\right|$ & $\begin{array}{l}6,47 \\
6,16 \\
6,16 \\
6,45\end{array}$ & $\begin{array}{l}-4,8 \\
-4,8 \\
-0,3\end{array}$ & $\begin{array}{l}208 \\
266 \\
262 \\
249\end{array}$ & $\begin{array}{l}+27,9 \\
+26,0 \\
+19,7\end{array}$ & $\left|\begin{array}{l}16,20 \\
16,48 \\
16,26 \\
15,91\end{array}\right|$ & $\begin{array}{r} \\
+1,7 \\
+0,4 \\
-1,8\end{array}$ \\
\hline 4 & 1,70 & $\begin{array}{c}\text { Vor d. Inj. } \\
\text { Nach } 3 \text { Min. } \\
\Rightarrow 15 \quad " \\
\Rightarrow 30 "\end{array}$ & $\begin{array}{l}200 \\
266 \\
262 \\
268\end{array}$ & $\begin{array}{r}+33,0 \\
+31,0 \\
+34,0\end{array}$ & $\left|\begin{array}{l}15,64 \\
15,64 \\
15,64 \\
15,31\end{array}\right|$ & $=\mid \begin{array}{l} \pm 0 \\
\pm 0 \\
-2,1\end{array}$ & $\begin{array}{l}5,83 \\
6,34 \\
5,86 \\
5,81\end{array}$ & $\begin{array}{l}+8,7 \\
+0,5 \\
+0,3\end{array}$ & $\begin{array}{l}193 \\
240 \\
249 \\
259\end{array}$ & $\begin{array}{r}+24,3 \\
+29,0 \\
+34,2\end{array}$ & $\begin{array}{l}15,64 \\
15,64 \\
15 ; 86 \\
15,29\end{array}$ & $\begin{array}{l} \pm 0 \\
+1,4 \\
-2,2\end{array}$ \\
\hline 5 & $\begin{array}{c}\hat{0} \\
1,50\end{array}$ & $\begin{array}{l}\text { Vor d. Inj. } \\
\text { Nach } 3 \text { Min. }\end{array}$ & $\begin{array}{l}186 \\
193\end{array}$ & $+3,8$ & $\begin{array}{l}18,20 \\
17,92\end{array}$ & $-1,5$ & $\begin{array}{l}5,43 \\
5,27\end{array}$ & $-2,8$ & $\begin{array}{l}184 \\
202\end{array}$ & $+9,8$ & $\begin{array}{l}17,92 \\
17,64\end{array}$ & $-1,6$ \\
\hline 6 & $\begin{array}{c}\hat{0} \\
1,65\end{array}$ & $\begin{array}{c}\text { Vor d. Inj. } \\
\text { Nach } 3 \text { Min. } \\
" 15 " \\
" 30 "\end{array}$ & $\begin{array}{l}172 \\
177 \\
217 \\
232\end{array}$ & $\begin{array}{r}+2,9 \\
+26,2 \\
+34,9\end{array}$ & $\left|\begin{array}{l}15,36 \\
15,36 \\
15,64 \\
14,80\end{array}\right|$ & $\begin{array}{c} \pm \\
\pm 0 \\
+1,8 \\
-3,6\end{array}$ & $\begin{array}{l}\mathbf{5}, 97 \\
\mathbf{5 , 9 7} \\
\mathbf{5 , 6 8} \\
\mathbf{5 , 6 8}\end{array}$ & $\begin{array}{l} \pm 0 \\
-4,8 \\
-4,8\end{array}$ & $\begin{array}{l}168 \\
199 \\
204 \\
199\end{array}$ & $\begin{array}{l}+18,4 \\
+21,4 \\
+18,4\end{array}$ & $\begin{array}{l}15,36 \\
15,36 \\
15,92 \\
15,24\end{array}$ & $\begin{array}{l} \pm 0 \\
+3,6 \\
-7,3\end{array}$ \\
\hline
\end{tabular}


wert herab, in anderen Versuchen zeigte ex allenfalls gegentiber dem Anfangswert erhöhte Werte, geringe Schwankung aufweisend, und zwar erwies sich der Zuckergehalt des Lebervenenblutes meistens als am höchsten (Fig. 4).

Aus obigen Daten ist also ersichtlich, dass im Peptonshock der Zuckerspiegel sich von dem Shockausbruch unmittelbar nachfolgender Zeit an erheblich erhöht ist und diese Erhöhung in drei Blutarten fast parallel geht, ferner dass der Zuckerspiegel, obschon er im halbstuindigen Ablauf in drei Blutarten mehr oder weniger Schwankungen unterworfen war, stets im Lebervenenblut den Maximalwert beibehält.

Hämoglobin: Unmittelbar nach Shockausbruch und im Ablauf von 15 Ninuten wurde im grossen und ganzen nur geringe Zunahme in drei Blutarten konstatiert, und diese Zunahme war im Lebervenenblut

4.

Eiweissgehaltes in der Leber beim Peptonshock.

\begin{tabular}{|c|c|c|c|c|c|c|c|c|}
\hline \multirow{2}{*}{\multicolumn{2}{|c|}{$\begin{array}{l}\text { Serumei- } \\
\text { weiss }\end{array}$}} & \multicolumn{6}{|c|}{ V. hepatica } & \multirow{3}{*}{ Bemerkungen } \\
\hline & & \multicolumn{2}{|c|}{ Zueker } & \multicolumn{2}{|c|}{$\mathrm{Hb}$} & \multicolumn{2}{|c|}{$\begin{array}{l}\text { Serumei- } \\
\text { weiss }\end{array}$} & \\
\hline$\%$ & $\begin{array}{l}\text { Diff. } \\
\text { in } \%\end{array}$ & $\mathrm{mg} /$ & $\begin{array}{l}\text { Diff. } \\
\text { in } \%\end{array}$ & $\mathbf{g} / \mathrm{dl}$ & $\begin{array}{l}\text { Diff. } \\
\text { in } \%\end{array}$ & \%á & $\begin{array}{l}\text { Diff. } \\
\text { in } \% 0\end{array}$ & \\
\hline $\begin{array}{l}6,14 \\
6,12 \\
5,77 \\
6,12\end{array}$ & $\begin{array}{l}-0,3 \\
-6,0 \\
-0,3\end{array}$ & $\begin{array}{l}215 \\
234 \\
236 \\
170\end{array}$ & $\begin{array}{r}+8,8 \\
+9,8 \\
-20,9\end{array}$ & $\begin{array}{l}14,39 \\
14,48 \\
14,52 \\
14,39\end{array}$ & $\begin{array}{l}+0,6 \\
+0,9 \\
\pm 0\end{array}$ & $\begin{array}{l}6,12 \\
5,90 \\
5,75 \\
5,77\end{array}$ & $\begin{array}{r}-3,6 \\
-6,0 \\
-5,7\end{array}$ & $\begin{array}{l}\text { 2 Min. nach Injektion Atmung leicht } \\
\text { beschleunigt. Kein Krampf. Shock } \\
\text { (土) }\end{array}$ \\
\hline $\begin{array}{l}6,21 \\
5,97 \\
5,97 \\
5,79\end{array}$ & $\begin{array}{l}-3,9 \\
-3,9 \\
-6,8\end{array}$ & $\begin{array}{l}193 \\
232 \\
232 \\
240\end{array}$ & $\begin{array}{l}+20,2 \\
+20,2 \\
+24,3\end{array}$ & $\begin{array}{l}14,76 \\
14,67 \\
14,76 \\
14,11\end{array}$ & $\begin{array}{l}-0,6 \\
\pm 0 \\
-4,4\end{array}$ & $\begin{array}{l}5,72 \\
5,79 \\
5,70 \\
5,79\end{array}$ & $\begin{array}{r}+1,2 \\
-0,3 \\
+1,2\end{array}$ & $\begin{array}{l}\text { a Min. nach Injektion beschleunigte } \\
\text { Atmung. Cyanose. Kein Krampf. } \\
\text { Shock }(+)\end{array}$ \\
\hline $\begin{array}{l}6,77 \\
6,34 \\
6,36 \\
6,27\end{array}$ & $\begin{array}{r}-6,3 \\
-6,0 \\
-7,4\end{array}$ & $\begin{array}{l}232 \\
278 \\
276 \\
272\end{array}$ & $\begin{array}{l}+19,8 \\
+19,0 \\
+17,2\end{array}$ & $\begin{array}{l}16,20 \\
16,48 \\
16,26 \\
15,91\end{array}$ & $\begin{array}{r}+1,7 \\
+0,8 \\
-1,8\end{array}$ & $\begin{array}{l}6,41 \\
6,38 \\
6,21 \\
6,34\end{array}$ & $\begin{array}{r}-0,5 \\
-3,1 \\
-1,1\end{array}$ & $\begin{array}{l}40 \text { Sekunden nach Injektion Krämpie. } \\
\text { Dauer } 20 \text { Sekunden. Shock (状) }\end{array}$ \\
\hline $\begin{array}{l}6,34 \\
6,38 \\
5,94 \\
5,92\end{array}$ & $\begin{array}{r}+0,6 \\
-6,3 \\
-6,6\end{array}$ & $\begin{array}{l}222 \\
260 \\
262 \\
260\end{array}$ & $\begin{array}{l}+17,1 \\
+18,0 \\
+17,1\end{array}$ & $\begin{array}{l}15,64 \\
15,64 \\
15,92 \\
15,29\end{array}$ & $\begin{array}{l} \pm 0 \\
+1,8 \\
-2,2\end{array}$ & $\begin{array}{l}6,16 \\
6,36 \\
5,90 \\
5,86\end{array}$ & $\begin{array}{r}+3,2 \\
-4,2 \\
-4,9\end{array}$ & $\begin{array}{l}40 \text { Sekunden nach Injektion Krämpfe. } \\
\text { Dauer } 30 \text { Sekunden. Shock (It) }\end{array}$ \\
\hline $\begin{array}{l}5,47 \\
4,85\end{array}$ & $-11,3$ & $\begin{array}{l}204 \\
222\end{array}$ & $+8,8$ & $\begin{array}{l}17,92 \\
18,20\end{array}$ & $+1,6$ & $\begin{array}{l}5,25 \\
5,27\end{array}$ & $+0,4$ & $\begin{array}{l}1 \text { Min. nach Injektion Krämple. } \\
\text { Min. nach Injektion Herzstillstand. }\end{array}$ \\
\hline $\begin{array}{l}6,03 \\
5,68 \\
5,81 \\
5,90\end{array}$ & $\begin{array}{r}-5,8 \\
-3,6 \\
-2,1\end{array}$ & $\begin{array}{l}181 \\
199 \\
251 \\
251\end{array}$ & $\begin{array}{r}+9,9 \\
+38,7 \\
+38,7\end{array}$ & $\left|\begin{array}{l}15,90 \\
15,36 \\
15,92 \\
14,80\end{array}\right|$ & $\begin{array}{l}+1,0 \\
+4,7 \\
-2,6\end{array}$ & $\begin{array}{l}5,92 \\
6,12 \\
5,72 \\
5,88\end{array}$ & $\begin{array}{r}+3,4 \\
-3,4 \\
-0,7\end{array}$ & $\begin{array}{l}\text { Nur beschleunigte Atmung. Kein } \\
\text { Krampf. Shock }(+)\end{array}$ \\
\hline
\end{tabular}




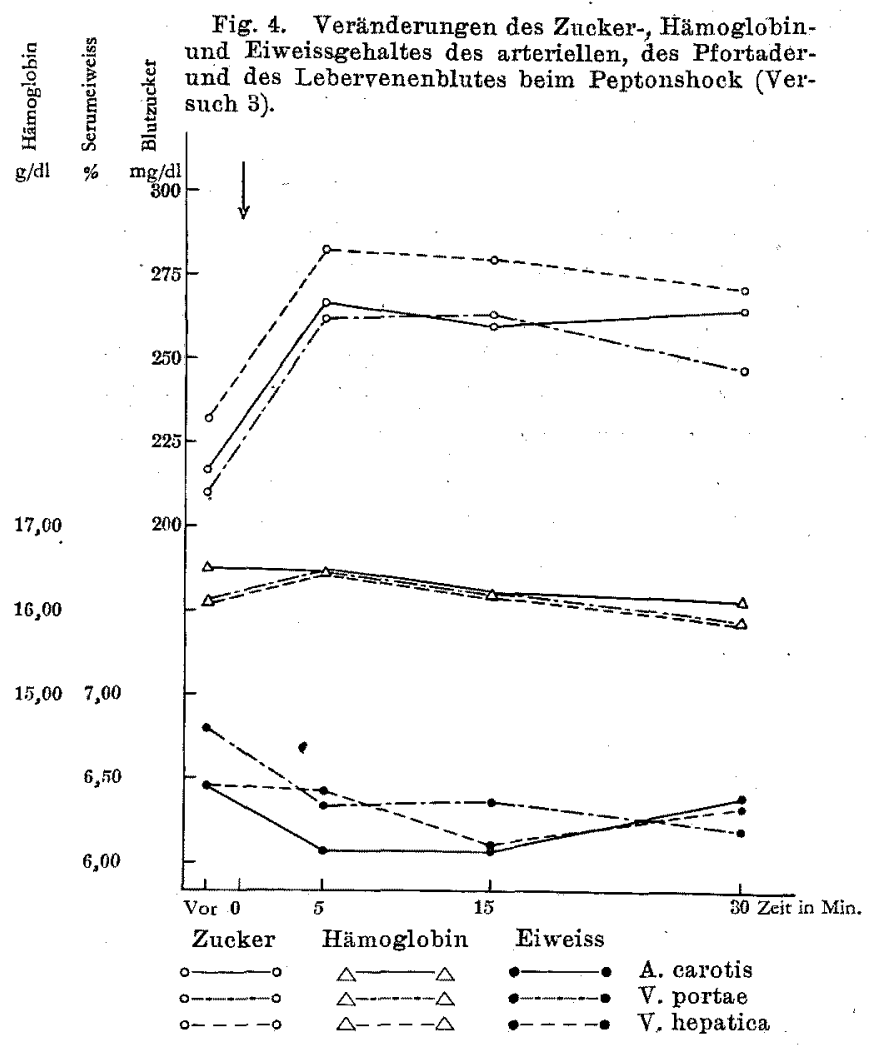

besonders deutlich. Nach 30 Minuten erfolgt die Abnahme in drei Blutarten, im Lebervenenblut ist dennoch die prozentische Abnahme gegenüber der Kontrolle geringer (Fig. 4).

Serumeiweiss: In direktem Anschluss an den Shockausbruch erfuhr der Eiweissgehalt des arteriellen Blutes, abgesehen davon, dass er in Versuch 4 zunahm und in Versuch 6 unverändert blieb, in allen iibrigen Versuchen Abnahme. Nach 15 Minuten fand in der Mehrzahl der Fälle noch weitere Abnahme statt, nach 30 Minuten erfuhr der vorher herabgesetzte Eiweisspiegel eine geringe Erhöhung. Im Pfortaderblut trat unmittelbar nach Shockausbruch eine Erhöhung nur in einem einzigen Versuch auf, in übrigen Versuchen wurde nur eine Erniedrigung beobachtet; nach 30 Minuten wechselten Erhöhung und Erniedrigung zur Hälfte miteinander ab. Im Lebervenenblut stellte sich unmittelbar nach Shockausbruch in Versuch 1, 3 und 4 eine Erniedrigung: 
ein, die aber gegenüber der Kontrolle geringergradig war, in anderen 3 Versuchen wurde eine geringe Erhöhung konstatiert. Nach $15 \mathrm{Mi}$ nuten traten Erniedrigungen in sämtlichen Versuchen ein, nach $30 \mathrm{Mi}$ nuten wurde wieder meistens die Tendenz zur Erhöhung beobachtet (Fig. 4).

Kurz zusammengefasst, im Peptonshock erhöht sich der Eiweissspiegel im Lebervenenblut unmittelbar nach Shockausbruch ein wenig; sinkt aber nachher mit dem Zeitablauf ab, während er im Pfortaderund arteriellen Blut von dem Shockausbruch unmittlelbar nachfolgender Zeit an abzusinken anfängt ; nach halbstündigem Ablauf erfährt der Eiweisspiegel in drei Blutarten, der inzwischen gemeinsam herabgesetzt worden war, geringe Erhöhungen. Derartige Veränderung des Eiweissspiegels im Peptonshock unterscheidet sich augenfällig von derselben im anaphylaktischen Shock, ist aber kaum verschieden von der Veränderung im Histaminshock.

\section{Besprechung.}

Recht umfangreich ist die Literatur über Veränderungen des Blutzuckers im Peptonshock. Nach Angaben von vielen Autoren scheint sich der Blutzucker hierbei je nach den Tierarten unterschiedlich zu verhalten. Die Angaben über Peptonwirkung auf den Zucker beim Hund lanten ziemlich widersprechend. Henderson $u$. Underhill ${ }^{54)}$ fanden in dem einen Fall die Hyper-, im andern Fall aber Hypoglykämie, Osato, ${ }^{36)}$ Achard u. Feuillié ${ }^{37)}$ sahen die Hyperglykämie auftreten. Im Gegensatz hierzu haben McGuigan u. Ross, ${ }^{39)}$ Mizuhara ${ }^{40)}$ die Hypoglykämie konstatiert; nach $\mathrm{K}$ at ${ }^{42}{ }^{42}$ tritt im Peptonshock zumeist Zunahme des Blutzuckers, aber auch gelegentlich $\mathrm{Ab}$ nahme auf. M. W at tan a b e ${ }^{38)}$ hat hervorgehoben, dass im Peptonshock als Grundsatz die Hyperglykämie auftritt, die aber nicht so hochgradig. ist und in 15-60 Minuten die Akme erreicht und nachher allmählich. abklingt. Indes geben eine Reihe von Forschern, wie z.B. Kuriya$\mathrm{ma}^{41)} \mathrm{Mizuhara},{ }^{40)} \mathrm{Kos}$ co e, ${ }^{94)} \mathrm{Okamoto}{ }^{17)} \mathrm{Kaiwa},{ }^{44)}$ und $\mathrm{H}$. Wa$\tan a b e^{45)}$ u. a. übereinstimmend an, dass bei Peptonkaninchen die Hyperglykämie auftritt.

Über dje Frage, durch welchen Wirkungsmechanismus das Pepton die Hyperglykämie zustande bringt, sind die Ansichten von verschiedenen Forschern sehr geteilt. Kato ${ }^{42}$ ist der Meinung, dass die Hyper-

04) Kosuge, Kyoto Igaklai Zasshi, 1924, 21, 1100. 
glykämie nach Pepton durch Insuffizienz der Leberfunktion bedingt sein dürfe, und dass die Veränderung des Blutzuckerspieg'els vom Fütterung'szustand des Tiers abhängig sei. Nakanis h ${ }^{43}$ hat der Meinung' Ausdruck gegeben, dass die Peptonhyperglykämie teils zentraler Natur, teils aber peripheren Ursprungs sei, indem das Pepton einerseits das Zuckerzentrum erregt, anderseits durch Hervorrufen von Staung in der Leber direkt das Leberglykogen mobilisiert. Kaiwa ${ }^{\mathbf{4 4})}$ ist der Ansicht, dass die Peptonhyperglykämie beim Hund durch peripheren Mechanismus bedingt sei, und dass beim Kaninchen die Aufrechterhaltung der: Splanchnici bis zu einem gewissen Grad auf die Hyperglykämie hemmend einwirkt. Brandes u. Simonds ${ }^{95}$ äusserten sich, dass im Peptonshock der Zucker des Lebervenenblutes erheblich zunimmt, während der Zuckergehalt venösen Blutes abnimmt.

In vorliegendem Versuchen kamen unmittelbar nach Shockausbruch Zuckererhöhungen im arteriellen, Pfortader- und Lebervenenblut beinahe parallel zum Vorschein; dadurch unterscheidet sich der Peptonshock vom anaphylaktischen Shock, zeigt aber nahezu gleiche Verhältnisse wie die im Histaminshock. Mit dem Zeitablauf sinkt der Zuckerspiegel in einigen Fällen ab, was auf dem Glykogenschwund beruhen dürfte.

Úber den Milchsäureumsatz im Peptonshock fehlt es meines Wissens bis dahin noch an Untersuchungen. $A b^{96)}$ hat den Nachweis erbracht, dass im Peptonshock arterieller Blutdruck absinkt, wohingegen im Pfortadergebiet, vor allem aber in der Leber hochgradige Stase zustande kommt. In meinen Versuchen trat der Milchsäureanstieg in dem Lebervenenblut am stärksten in den Vordergrund, das dürfte dahin gedeutet werden, dass infolge der Stase in der:Leber die Glykogenolyse sich in erhöhtem Masse vollzieht und zu vermehrter Milchsäurebildung: führt, wobei auch durch Störungen der Leberfunktion die Milchsäureresynthese gestört wird. Die Milchsäureanhäufung im Pfortaderblut duirfte wohl auch auf die Stase durch die Zirkulationsstörung im Pfortadergebiet zurückzuführen sein.

Es erhebt sich nun die Frage: Woher kommt es, dass, während im anaphylaktischen und Histaminshock unmittelbar nach Shockausbruch der Milchsäuregehalt im arteriellen Blut, wenn auch vorübergehend, erheblich abnimmt, im Peptonshock hingegen der Milchsäuregehalt fast unverändert bleibt? Mautner u. Pick ${ }^{87}$ fanden im Peptonshock bei Kaninchen eine Verengerung der Lungengefässe; $A b^{61)}$ hat her-

95) Brandes u. Simonds, Amer. Journ. Physiol, 1928, 86, 618.

96) A be, Tohoku Igaku Zasshi, 1919, 4, 500. 
vorgehoben, dass Pepton bei Kaninchen, indem es einerseits auf die Iungenarterien selbst, andererseits aber auch auf die Bronchialmuskulatur einwirkt, diese zur Konstriktion bringt und auf diese Weise direkt und indirekt das Kaliber der Lungenarterien einengt und dadurch den Lungenkreislauf stört. Nolf $f^{97}$ hat am Hund nachgewiesen, dass Pepton die Konstriktion kleiner Arteriolen der Lunge herbeiführt. Indes hat in jüngster Zeit $\mathrm{Sato}^{68}$ ) darüber berichtet, dass im Peptonshock beim Kaninchen die Verengerung der Lungenarterien röntgenologisch nicht so deutlich ist wie im anaphylaktischen und Histaminshock. Im Peptonshock dürfte die Störung des Lungenkreislaufes, folglich auch der Sauerstoffmangel in der Lunge weniger stark sein, wie in andern beiden Shockarten, so dass die oxydative Milchsäurebeseitigung nicht so lebhaft wie in anderen beiden Shockarten vonstatten gehen würde, was wahrscheinlich das Ausbleiben der Herabsetzung des arteriellen Milchsäurespiegels bedingt (Siehe I. Mitteilung ${ }^{\text {1) }}$ vorliegender Studien). Weil aber auch im Peptonshock die Verengerung der Lungengefässe und die Kontraktion der Bronchialmuskulatur mehr oder weniger sich geltend machen, kann die Störung des Lungenkreislaufes in wenn auch mässigem Grad, zustande kommen, wodurch die intrapulmonale oxydative Milchsäurebeseitigung uiber die Norm hinaus vor sich geht. In Hinsicht darauf dürfte die scheinbar paradoxe Erscheinung, dass der Milchsäurespiegel arteriellen Blutes durch die Milchsäure, die unter dem Shock in überaus vermehrtem Masse aus Lebervenen und anderem venösen System in die allgemeine Zirkulation eingedrungen ist, sonst erhöht gefunden werden sollte, unter Umständen umgekehrt eher absinkt, sich erklären lassen.

Dass im Peptonshock Bluteindickung und Serumeiweissabnahme zum Vorschein kommt, ist schon fruher seit Untersuchungen von $\mathrm{He}$ denhain ${ }^{46)}$ und Starling ${ }^{47)}$ allgemein bekannte Tatsache; über ihre Entstehungsmechanismen sind dennoch die Ansichten sehr verschieden geteilt, indem eine Reihe Autoren die Reaktion der Leber, andere den kapillären Ursprung annehmen und wieder andere eine vermittelnde Anschauung hervorheben. Allein soviel scheint festzustehen, dass die Peptonwirkung je nach der Individualität der Tiere verschieden empfunden wird, und dass bei Fleischfressern, besondens beim Hund Bluteindickung und Serumeiweissabnahme als typisch gelten. Heidenhain injizierte einem Hund Pepton intravenös und sah vermehrten Lymphabfluss aus dem Ductus thoracicus; Starling hat den Beweis

97) Nolf, Rev. belge. Sci. méd., 1931, 3, 697. 
gefüht, dass die aus dem Ductus thoracicus fliessende Lymphe von der Leber herruhrt. $\mathrm{Abe}^{95)}$ ist der Neinung; dass die lymphbildende Wirkung von Pepton durch Stase in den Organen bedingt ist und dass der Grund für vornehmlichen Abfluss der gebildeten Lymphe aus' der Leber in sehr erheblichen Stase in der gelegen ist. Simond $\mathrm{s}^{\mathbf{9})}$ hat im Peptonshock des Hundes betächtliche Erhöhung der Blutkonzentration konstatiert. Saito u. Nakazaw $a^{99)}$ an hiesiger Klinik fanden ebenso auch an Hunden neben der Zunahme des Lymphausflusses eine Abnahme des Serumeiweisses konstatiert und beides auf die Permeabilitätssteigerung des Gefässendothels zurückgeführt. Tera.o ka ${ }^{27}$ ) fand auch am Hund einen Hämoglobinanstieg und eine Serumeiwieissabnahme.

Nach Ansicht der meisten Autoren soll das Kaninchen, der Pflanzenfresser, im Gegensatz zum Hund gegen das Pepton weniger empfindlich sein, sodass bei diesem Tiere die Peptonwirkung nicht sicher zum Vorschein kommt. Sato ${ }^{49}$ berichtete, dass im Peptonshock des Kaninchens, selbst wenn anscheinend keine Shocksymptome ausfindig: gemacht werden können, erhöhte Blutkonzentration anzutreffen ist.

In vorljegenden Versuchen wurde von dem Shockausbruch unmittelbar nachfolgender Zeit an bis zum Ablauf von Viertelstunde eine, wenn auch geringfügige Bluteindickung festgestellt. Der Grad dieser Bluteindickung war im Lebervenenblut um ein Geringes stärker als in anderen zwei Blutarten. Seiumeiweiss nahm im arteriellen und Pfortaderblut in beinahe gleichem Masse wie bei der Kontrolle ab, so dass es fast als unverändert angesehen werden konnte. Da indessen im Lebervenenblut eine geringe Zunahme des Serumeiweisses angetroffen wurde, liegt die Annahme nahe, dass das Pepton wohl auch bei Kaninchen die Leber aufs stärkste angreift und wie viele Autoren angeben, die Permeabilitätssteigerung des Lebergefässendothels hervorrufen, wodurch die Leberzellen ödematös anschwellen und Wasser an sich ziehen, infolgedessen es im Lebervenenblut zu relativ stärkerer Bluteindickung kommen dürfte.

Aus der Tatsache, dass, obwohl die Zu- und Abnahme des Hämoglobin- und Eiweissgehaltes im Lebervenenblut individuell verschieden resultiert, doch die Zunahme des Eiweisses in der Hälfte der sämtlichen Versuche im Vergleich mit derselben des Hämoglobins grösser ist, kann man sich leicht die Vorstellung konstruieren, dass ein Teil des Vorrateiweisses im Lebergewebe in die Lebervenen hinein mobilisiert

98) Sim o nds, Amer. Journ. Physiol., 1925, 72, 1.

99) Saito u. Nakazawa, Tohoku Journ. Exp. Med., 1932, 19, 233. 
wird. Allein diese Eiweissmobilisation ist jedoch geringfügig und zudem transitorischer Natur, was sich augenscheinlich vom Verhalten beim anaphylaktischen Shock unterscheidet, aber mit dem beim Histaminshock in naher Beziehung steht.

\section{Zusammenfassung.}

Wenn man beim Kaninchen den Histamin- oder Peptonshock ausgelöst hat, so tritt im arteriellen Blut bei der ersten Shockform unmittelbar nach Shockausbruch eine temporäre Milchsäureabnahme auf, während in der letzteren Shockform hingegen der arterielle Milchsäurespiegel fast unverändert bleibt; mit dem Zeitablauf nimmt die Milchsäure bei beiden Shockarten allmählich zu.

Im Pfortader- und Lebervenenblut erhöht sich der Milchsäurespiegel in beiden Shockarten von direktem Anschluss an den Shockausbruch an, besonders ausgeprägt ist diese Erhöhung im Lebervenenblut.

Zuckergehalt nimmt in beiden Shockarten in drei Blutarten nahezu paralell zu; nachher erhöht sich der Zuckerspiegel allmählich immer stärker, sinkt aber mitunter eher ab, allerdings wird der Ursprungswert noch überschritten.

Hämoglobingehalt vermehrt sich in beiden Shockformen in drei Blutarten von dem Shockausbruch unmittelbar nachfolgender Zeit an bis zum Ablauf von Viertelstunde; insbesondere im Lebervenenblut übertrifft der Hämoglobinswert um ein Geringes Werte in anderen 2 Blutarten. Mit dem Zeitablauf treten allmähliche Abnahme in 3 Blutarten ein.

Serumeiweissgehalt zeigt in beiden Shockformen im arteriellen und Pfortaderblut gleichgradige Abnahme wie die bei der Kontrolle, im Lebervenenblut hingegen eine geringe Zunahme. Nachher treten in der Mehrzahl der Fälle graduelle Abnahmen in drei Blutarten in Erscheinung.

Wenn man oben angeführte Veränderungen in beiden Shockformen mit Veränderungen im anaphylaktischen Shock (I. Mitteilung) vergleicht, so erkennt man, dass hinsichtlich des Blutzucker- und Eiweissumsatzes ein markanter Unterschied zwischen jenem und diesem Shock besteht. Vor allem aber bezüglich des Eiweissumsatzes ist die Reagierbarkeit der Leber hier bei weitem grösser als im Histaminund Peptonshock.

Was die Blutmilchsäure anblangt, so zeigt beim Peptonshock der Milchsäurespiegel, der im anaphylaktischen und Histaminshock 
unmittelbar nach Shockausbruch transitorisch erhebliche Erniedrigungen erfährt, keine Spur von derartiger Erscheinung. Das dürfte wahrscheinlich dahin gedeutet werden, dass die intrapulmonale Milchsäureverbrennung, während sie im anaphylaktischen und Histaminshock lebhaft vonstatten geht, im Peptonshock nicht so in den Vordergrund, wie in ersteren beiden Shockformen, tritt. 